ARTICLE

DOI: $10.1038 / \mathrm{s} 41467-017-02501-4$

\title{
Streamlined sensory motor communication through cortical reciprocal connectivity in a visually guided eye movement task
}

Takahide Itokazu1, Masashi Hasegawa1, Rui Kimura1, Hironobu Osaki17, Urban-Raphael Albrecht', Kazuhiro Sohya ${ }^{2}$, Shubhodeep Chakrabarti (1) ${ }^{1}$, Hideaki Itoh ${ }^{3}$, Tetsufumi Ito ${ }^{4}$,

Tatsuo K. Sato ${ }^{1,5} \&$ Takashi R. Sato ${ }^{1,2,6}$

Cortical computation is distributed across multiple areas of the cortex by networks of reciprocal connectivity. However, how such connectivity contributes to the communication between the connected areas is not clear. In this study, we examine the communication between sensory and motor cortices. We develop an eye movement task in mice and combine it with optogenetic suppression and two-photon calcium imaging techniques. We identify a small region in the secondary motor cortex $\left(\mathrm{MO}_{\mathrm{s}}\right)$ that controls eye movements and reciprocally connects with a rostrolateral part of the higher visual areas $\left(\mathrm{V}_{\mathrm{RL} / \mathrm{A} / \mathrm{AL}}\right)$. These two regions encode both motor signals and visual information; however, the information flow between the regions depends on the direction of the connectivity: motor information is

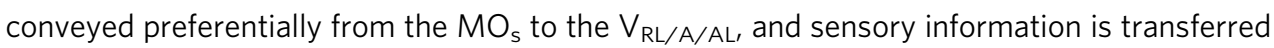
primarily in the opposite direction. We propose that reciprocal connectivity streamlines information flow, enhancing the computational capacity of a distributed network.

\footnotetext{
${ }^{1}$ Werner Reichardt Center for Integrative Neuroscience, University of Tübingen, 72076 Tübingen, Germany. ${ }^{2}$ Department of Mental Disorder Research, National Institute of Neuroscience, National Center of Neurology and Psychiatry, Tokyo 187-8502, Japan. ${ }^{3}$ Graduate School of Science and Engineering, Saga University, Saga 840-8502, Japan. ${ }^{4}$ Department of Anatomy, Kanazawa Medical University, Ishikawa 920-0293, Japan. ${ }^{5}$ Institute of Neuroscience, Technical University of Munich, 80802 Munich, Germany. ${ }^{6}$ PRESTO Japan Science and Technology Agency, Saitama 332-0012, Japan. ${ }^{7}$ Present address: Department of Physiology, Tokyo Women's Medical University, Tokyo 162-8666, Japan. Takahide Itokazu, Masashi Hasegawa and Rui Kimura contributed equally to this work. Correspondence and requests for materials should be addressed to T.K.S. (email: tatsuo.sato@tum.de)

or to T.R.S. (email: takashi.sato@cin.uni-tuebingen.de)
} 
T he cerebral cortex contains many anatomically defined areas ${ }^{1}$, each of which is proposed to have its preferred functional specialization ${ }^{2}$. These areas integrate with each other through long-range connections to perform complex yet efficient cortical computations ${ }^{3}$. For sensory processing, these connections link separate cortical areas and allow them to operate as a distributed hierarchical network ${ }^{2,4-6}$. Although the wiring patterns behind these connections have been subject to intensive investigation $^{7,8}$, genetical and optical approaches have only just enabled us to tackle information flow through long-range connectivity in the sensory cortex in vivo ${ }^{9-13}$.

Long-range reciprocal connectivity also links sensory and motor cortical areas, thus forming sensory-motor cortical circuits. These circuits have been extensively studied in rodent whisker systems on an anatomical and physiological level ${ }^{11-19}$. Rather than processing information independently (Supplementary Fig. 1a), sensory and motor areas are tightly coupled to process two fundamentally different signals: externally evoked sensory information and internally generated motor information. The notion of processing two different signals in a distributed network is supported by the existence of motor signals in sensory areas and sensory signals in motor areas. For instance, sensory stimulation causes depolarization in the motor cortex due to long-range connections between the somatosensory and motor cortices $^{14}$. Similarly, connections from the motor cortex can enhance sensory responses in the somatosensory cortex ${ }^{15}$. This overlapping network of sensory and motor signals raises a fundamental question of what information is communicated between these two cortical areas. One possibility is that the connectivity operates as a recurrent loop in which similar information is sent back and forth in both directions (Supplementary Fig. 1b), analogous to signal amplification mechanisms proposed in recurrent local networks ${ }^{20,21}$. Another possibility is that conveyed information is direction dependent and streamlined; in this case, the sensory cortex would select only sensory information from intermixed signals to send to the motor cortex, and the motor cortex would select only motor information to convey back to the sensory cortex (Supplementary Fig. 1c). Such directiondependent flow of information might contrast with the extensive interactions and similarities between sensory and motor cortices but could increase the computational capacity as has been proposed in generative models of hierarchical cortical processing 4,22 .

To distinguish between these two possibilities, we investigated sensory-motor network activity in mice undergoing a visually guided eye movement task. By employing this task, we identified a small region in the secondary motor cortex $\left(\mathrm{MO}_{\mathrm{s}}\right)$ that controls eye movements and found that this area was reciprocally connected to a rostrolateral part of the higher visual areas $\left(\mathrm{V}_{\mathrm{RL} / \mathrm{A} / \mathrm{AL}}\right)$. These sensory and motor areas encoded both sensory information and motor signals, implying that they operate as a distributed network. However, the connections from the sensory to the motor area primarily conveyed sensory information and those from the motor to the sensory area preferentially carried motor signals. Our results provide insight into how reciprocal connectivity operates in a distributed network of sensory-motor interactions.

\section{Results}

A visually guided eye movement task in mice. Despite their significance in the visual system, visually guided eye movements have been little studied in mice. We developed a behavioral task in which mice first had to identify an external visual stimulus as sensory input and then generate eye movements as motor output. In this task, we trained head-fixed mice to direct their left eye toward a central fixation light-emitting diode (LED) (Fig. 1a, b and Supplementary Fig. 2). Within 750-1000 ms, we turned on one of the two target LEDs (nasal or temporal) to instruct the mice which direction they should move their eye. If the mice successfully shifted their left eye in the direction of the target LED, we rewarded them with a drop of water. After several weeks of training, mice became capable of performing eye shifts (Fig. 1c, d) with a success rate of $85.9 \pm 2.2 \%$ and a reaction time of $772 \pm$ $118 \mathrm{~ms}$ ( $n=19$ mice). During the task, the movement of other body parts, such as the forelimbs or whiskers, did not correlate with eye movements or the onset of the visual target (Supplementary Fig. 3).

Unexpectedly, even though the task only required movement of the left eye, both eyes moved together as a coupled movement (Fig. 1e and Supplementary Movie 1). When the left eye moved toward the nasal side (solid cyan trace in Fig. 1e), the right eye moved toward the temporal side (dotted cyan trace); when the left eye moved toward the temporal side (solid magenta), the right eye moved toward the nasal side (dotted magenta). The movements of the two eyes were highly synchronized, although the amplitudes and peak velocities were smaller for temporal than nasal eye movements (amplitude: $13.5 \pm 0.8$ vs. $8.8 \pm 0.4$ degrees; velocity: $1019 \pm 46$ vs. $498 \pm 23$ degrees/s; Fig. 1f, g, Supplementary Fig. 4; both eyes filmed for 10 mice).

The eye movements that we observed were qualitatively similar to saccades in primates albeit with some notable differences. First, the reaction time $(772 \pm 118 \mathrm{~ms})$ was longer compared to that in macaques $^{23}$ and humans ${ }^{24}(\sim 200 \mathrm{~ms})$. Second, the peak velocity of the eye movements was larger than that of macaque ${ }^{25}$ and human ${ }^{26}$ saccades with similar amplitudes ( 300 degrees/s for 10 degrees saccades). Third, the post-eye movement drifts were larger than in primates ${ }^{27}$. Finally, in mice the nasal eye movements were larger than the corresponding temporal eye movements, but the reverse is true in humans ${ }^{28}$. Despite these differences, the binocular coupling of voluntary eye movements suggests that the mouse brain implements basic circuits for binocular eye movements, similar to those in foveate mammals.

Secondary motor cortex is required for eye movement task. We investigated whether the motor cortex was required for the eye movements during our behavioral task by performing optogenetic suppression. As a first step toward optogenetic suppression, we identified a candidate region involved in eye movement. We performed systematic microstimulation in a medial portion of the frontal cortex. Low-current microstimulation (up to $50 \mu \mathrm{A}$ ) in awake mice evoked binocularly coupled rapid eye shifts (Supplementary Fig. 5a, b) when the stimulation was in a caudomedial portion of the frontal cortex $(\sim 700 \mu \mathrm{m}$ depth; $\sim 0.7 \mathrm{~mm}$ rostral and $\sim 0.7 \mathrm{~mm}$ lateral to the bregma; Supplementary Fig. $5 \mathrm{c}-\mathrm{e}$ ). The same stimulation current could also evoke whisker movements in a wide region of the frontal cortex as previously reported ${ }^{17}$. A much stronger current (up to $200 \mu \mathrm{A}$ ) could elicit eye movements reliably even in other areas, such as the anterior cingulate $\operatorname{area}^{29}$ (data not shown). In subsequent experiments using optogenetics and imaging, we targeted the coordinate $\sim 0.7$ $\mathrm{mm}$ lateral and $\sim 0.7 \mathrm{~mm}$ rostral to the bregma; this part of frontal cortex was free of large vessels, reliably elicited eye movements as large as those seen during the task (Supplementary Fig. 5e), anatomically projected to eye-movement related areas of the midbrain (Supplementary Fig. 6), and was located within the secondary motor cortex, a broadly defined area in the frontal $\operatorname{cortex}^{7}$ (Supplementary Fig. $5 c-0$ ). In this study, the region at this coordinate is referred to as the $\mathrm{MO}_{s}$.

To examine whether the $\mathrm{MO}_{s}$ is necessary for voluntary eye movements, we optogenetically suppressed neural activity 
a

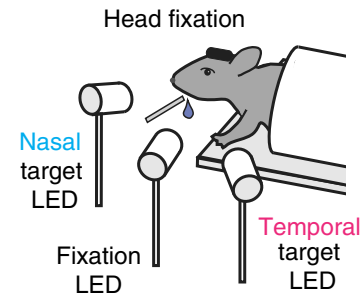

b

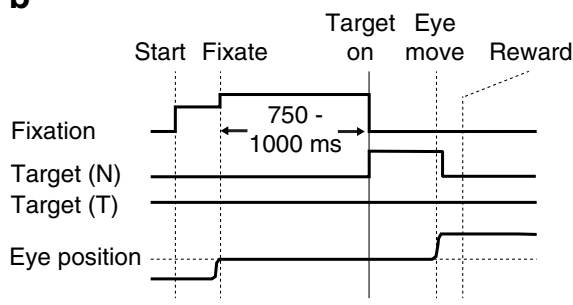

d

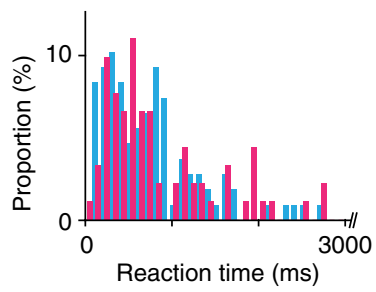

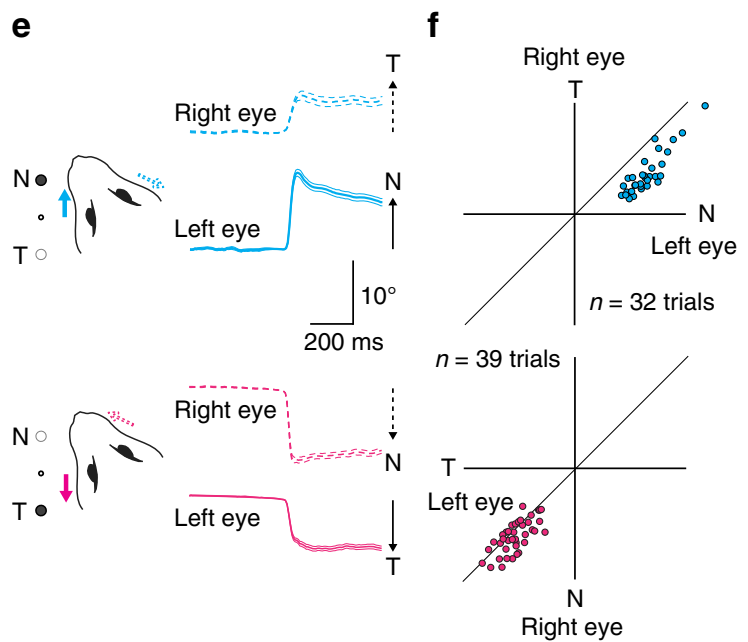

g
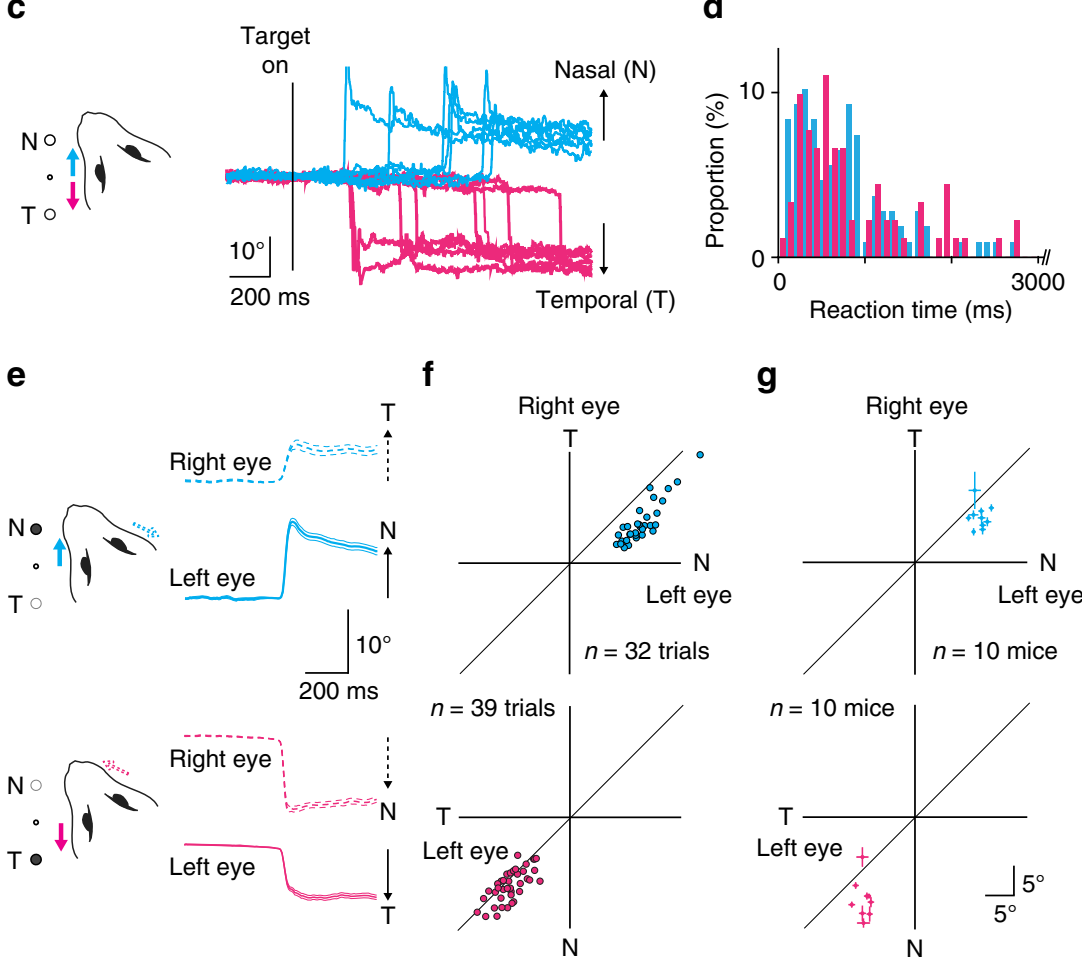

Right eye

Fig. $1 \mathrm{~A}$ visually guided eye movement task in mice. a The experimental design. Three LEDs instructed when and where to move the left eye. $\mathbf{b}$ After the mice fixated the central LED, one of the two LED targets (nasal or temporal) was turned on, and mice were required to shift their left eye toward the target. c Representative eye traces recorded during one behavioral session. Traces are aligned to the target onset. Cyan traces, trials with nasal target; magenta traces, trials with temporal target. $\mathbf{d}$ The distributions of reaction times for one animal (cyan, nasal target condition; magenta, temporal target condition). Reaction time range $<3 \mathrm{~s}$ (nasal 91.7\%; temporal 81.3\%) is shown because of the long tail of its distributions. e Average traces for eye movement for both eyes in one behavioral session (nasal, 32 trials, cyan; temporal, 39 trials, magenta). Traces for the left eye, solid lines; right eye, dotted lines. Thick and thin lines represent mean \pm s.e.m. $\mathbf{f}$ The amplitude of the eye movements for the left and right eyes (cyan, nasal target; magenta, temporal). The same animal as in $\mathbf{e . ~} \mathbf{g}$ The average eye movement amplitude is shown for the nasal (cyan) or temporal (magenta) target trials in 10 mice. Error bars indicate s.e.m

in this region during the eye movement task. Specifically, we locally activated parvalbumin (PV) interneurons that expressed channelrhodopsin-2 (ChR2) via viral transduction ${ }^{30,31}$ (Fig. 2a-c). The optogenetic suppression impeded contraversive movements severely (Fig. $2 \mathrm{~d}, \mathrm{f}, \mathrm{h}, \mathrm{PV}$ activation, $n=96$ trials; control, $n=88$ trials; $p<10^{-11}$, Pearson's chi-square test) but had only minor effects on ipsiversive movements (i.e., toward the ipsilateral side, $\mathrm{PV}$ activation, $n=84$ trial; control, $n=126$ trials; $p=0.0501$, Fig. 2e, g, i). In contrast, optogenetic suppression of the primary motor cortex, $\sim 1 \mathrm{~mm}$ away from $\mathrm{MO}_{s}$, did not affect eye movements (Supplementary Fig. 7). These results suggest that the $\mathrm{MO}_{\mathrm{s}}$ but not primary motor cortex is critical for visually guided eye movements, particularly contraversive ones.

The $\mathrm{MO}_{\mathrm{s}}$ encodes both motor signals and visual information. We predicted that the cortical area that controls eye movements would show neural activity related to the motor commands. To confirm this prediction, we investigated the neural activity of layer 2/3 neurons in the $\mathrm{MO}_{s}$ of head-fixed mice while they performed the eye movement task (Fig. 3a). We imaged fluorescent signals of the virally expressed, genetically encoded calcium indicator GCaMP6f as a readout for neural activity ${ }^{32}$ (Fig. 3b-d, Supplementary Fig. 5o). To help separate motor signals from the visual response, we rewarded mice for performing eye movements with a longer reaction time (Supplementary Fig. 8) and analyzed only trials with longer reaction times $(>1.5$ s). Twelve percent of neurons (471 out of 3787) showed an increase in fluorescent signals prior to the onset of eye movements (Fig. 3d-f). Previous studies in macaques considered neural activity preceding the onset of the eye movements to represent motor commands ${ }^{23,33-35}$; therefore, we will refer to this neural activity as motor activity. In contrast, neural activity after the onset of eye movements is unlikely to represent the motor command, and rather may be a mixture of visual activity, residual motor activity, and post-movement motor-related activity ${ }^{36}$. Across the population of neurons, motor activity showed a preference for the contraversive movement condition (Fig. 3h, j, $n=$ $471, p<10^{-12}$, Wilcoxon signed-rank test), consistent with the 
a

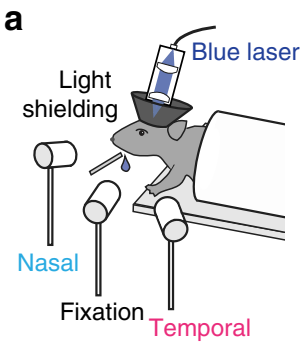

b

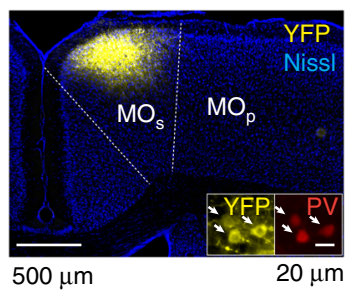

d

Contraversive movements

Control

PV activation<smiles>C1CCC2CCOC2CC1</smiles>

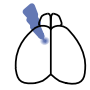

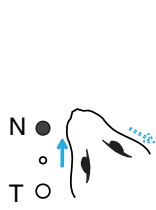

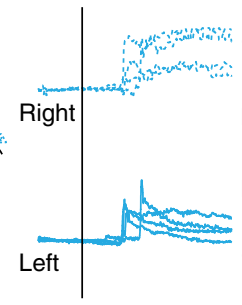

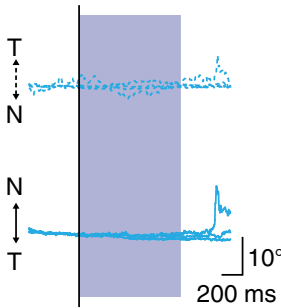

f<smiles>C1C2CC1C2</smiles>

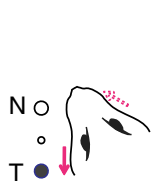
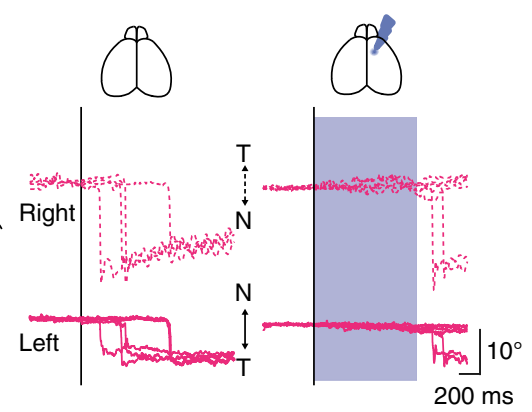

C

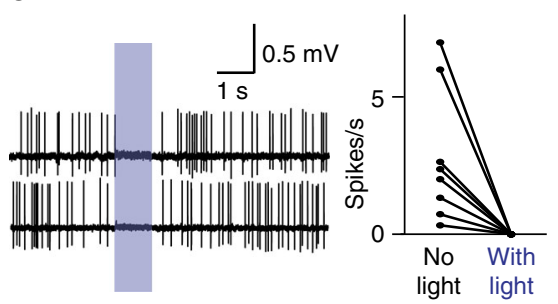

e Ipsiversive movements Control PV activation<smiles>C1CCC2OCCC2C1</smiles><smiles>O=C1CCC2CCCCC12</smiles>

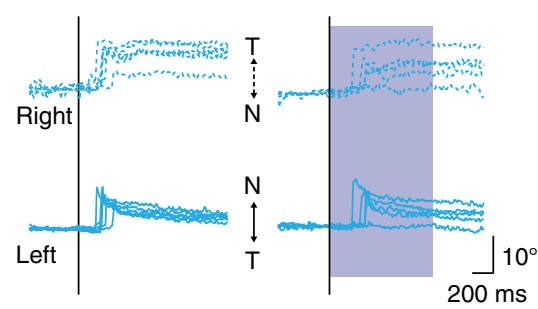

g

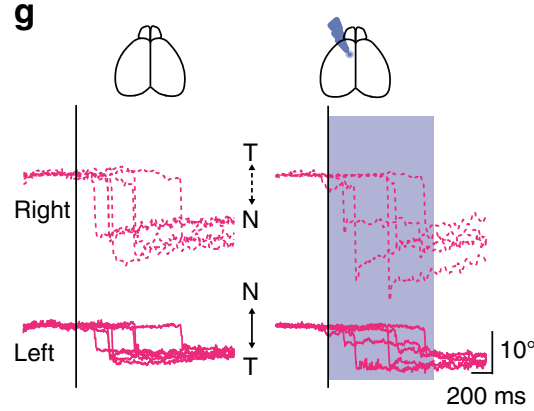

h

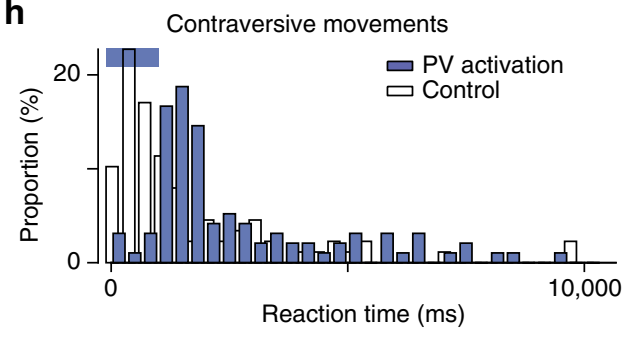

i

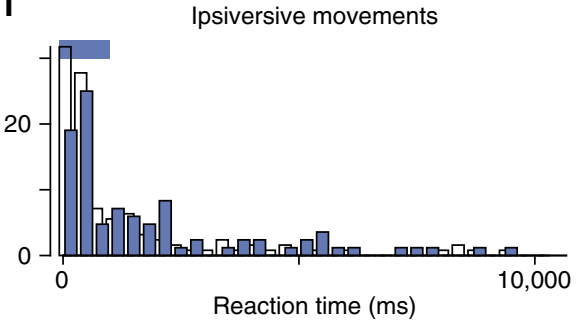

Fig. 2 Optogenetic suppression of the $\mathrm{MO}_{\mathrm{s}}$ during the visually guided eye movement task. a The experimental design. $\mathbf{b}$ ChR2 was expressed virally in PV interneurons in Pvalb-IRES-Cre transgenic mice. AAV-DIO-ChR2-eYFP was injected into the $\mathrm{MO}_{\mathrm{s}}$ (coronal section stained with Nissl), and ChR2-eYFP expression was confined to cells expressing PV (white arrows). c Firing was suppressed during blue light illumination. Left, two representative neurons are shown. Right, blue light illumination completely suppressed firing activity in all eight neurons $(p<0.01$, Wilcoxon signed-rank test). The activity was monitored with blind cell-attached recording. $\mathbf{d}$-g Optogenetic suppression of the $\mathrm{MO}_{\mathrm{s}}$ during the task. The virus was injected into the $\mathrm{MO}_{\mathrm{s}}$ either in the left $(\mathbf{d}, \mathbf{g})$ or right $(\mathbf{e}, \mathbf{f})$ hemisphere. Unilateral suppression of the $\mathrm{MO}_{\mathrm{s}}$ severely impaired eye movements in the contraversive (d, $\left.\mathbf{f}\right)$ but only mildly in the ipsiversive $(\mathbf{e}, \mathbf{g})$ direction. $\mathbf{h}, \mathbf{i}$ Reaction times for the eye movements. PV activation delayed the onset of contraversive eye movements $(\mathbf{h}, \mathrm{PV}$ activation, $n$ $=96$ trials; control, $n=88$ trials; $p<10^{-11}$ ), but showed only minor effects on ipsiversive movements (i, PV activation, $n=84$ trial; control,

$n=126$ trials; $p=0.0501$, Person's chi-square test)

role of the $\mathrm{MO}_{\mathrm{s}}$ in contraversive eye movements. The preference started $250 \mathrm{~ms}$ prior to the onset (Supplementary Fig. $9 \mathrm{f}-\mathrm{j}$ ).

The $\mathrm{MO}_{\mathrm{s}}$ also encoded visual information to instruct the eye movements. Fourteen percent of neurons (519 out of 3787) showed an increase in fluorescence signal immediately after the presentation of a visual target (Fig. 3d-f). Previous studies reported visual activity in this region just after visual stimulation in anesthetized or awake quiet mice 37,38 ; therefore, we will refer to this activity as visual activity. Consistently, we found that this activity was not significantly affected by the presence or absence of eye movements (Supplementary Fig. 10) and showed a slight preference for contraversive movement (Fig. 3g, i; $n=519, p<$ 0.05 , Wilcoxon signed-rank test). The preference was relatively stable at least up to $800 \mathrm{~ms}$ after the onset (Supplementary Fig. 9a-e). Taken together, our in vivo calcium imaging experiments demonstrate that the neurons in the $\mathrm{MO}_{\mathrm{s}}$ represent visual information immediately after the visual target and motor signals just before movement onset. 
a

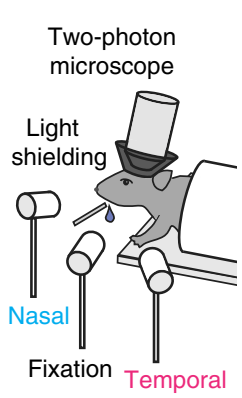

b

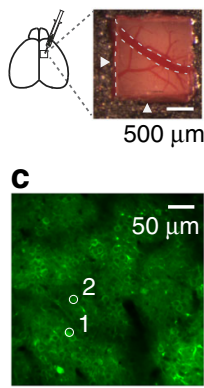

e

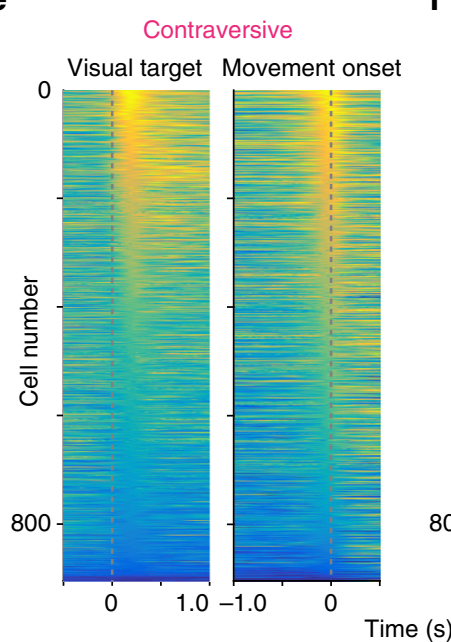

d

Visual target Movement onset
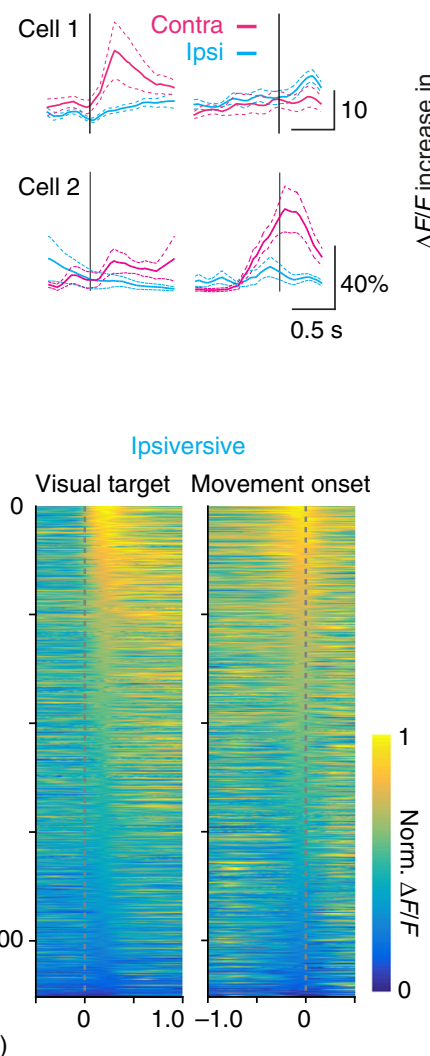

g

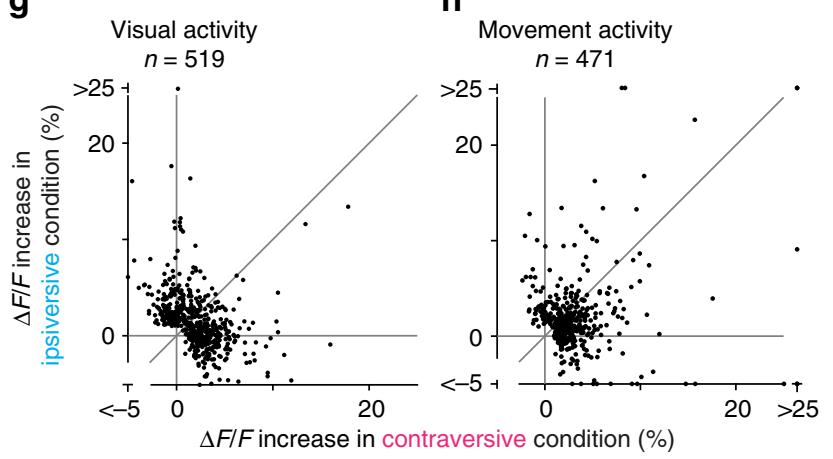

i

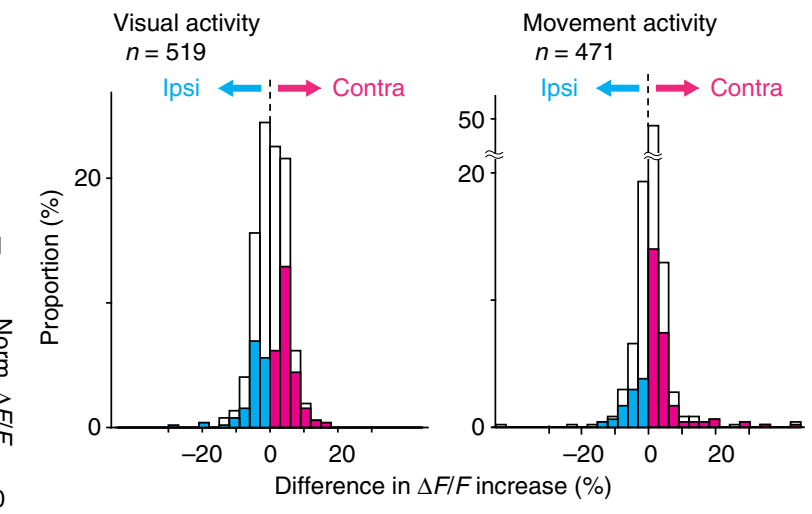

Fig. 3 Neural coding in the $\mathrm{MO}_{\mathrm{s}}$. a The experimental design. b Cranial window for two-photon imaging. Arrowheads indicate $700 \mu \mathrm{m}$ rostral and $700 \mu \mathrm{m}$ lateral from the bregma. Note a bridging vein leading to the superior sagittal sinus, which often runs around the coordinates. $\mathbf{c}$ Virally expressed GCaMP6f in the $\mathrm{MO}_{\mathrm{s}}$ for two-photon calcium imaging during the visually guided eye movement task. $\mathbf{d}$ Averaged fluorescence change of two example cells (ROIs shown in c, aligned to the onset of the visual target or to the onset of the eye movement; cyan, the trial condition for ipsiversive eye movement; magenta, for contraversive). e, f Sorted normalized $\Delta F / F$ traces for 904 neurons showing significant response either to visual targets or to eye movements. Response was aligned to the onset of the visual target or the movement in the contraversive (e) or ipsiversive (f) trials. $\mathbf{g}$-j Comparison between contraversive and ipsiversive movement trials for visual and motor activity ( $n=519$ or 471 neurons, showing significant visual or motor activity). $\mathbf{i}, \mathbf{j}$ A histogram for difference between ipsiversive and contraversive conditions in visual or motor activity. Neurons with a larger response in the contraversive condition ( $p<$ 0.05, Mann-Whitney test) were labeled as magenta ( $n=135$ in visual activity, 122 in motor activity) and neurons with a larger response in the ipsiversive condition in cyan $(n=80,45)$. The population preferred for the contraversive condition in visual activity $(n=519, p<0.05$, Wilcoxon signed-rank test $)$ and in motor activity $\left(n=471, p<10^{-12}\right)$

The $\mathrm{MO}_{\mathrm{s}}$ is reciprocally connected with visual areas RL/A/AL. The encoding of visual information in the $\mathrm{MO}_{\mathrm{s}}$ implies that the $\mathrm{MO}_{\mathrm{s}}$ interacts extensively with the visual cortex through longrange anatomical connections. Indeed, by labeling axon terminals of the $\mathrm{MO}_{\mathrm{s}}$ with tdTomato (Fig. 4), we found that the $\mathrm{MO}_{\mathrm{s}}$ projects to parieto-occipital regions of the cortex, including the visual cortex $(\sim 2.0 \mathrm{~mm}$ posterior and $\sim 2.5 \mathrm{~mm}$ lateral to the bregma). To identify to where in the visual cortex the $\mathrm{MO}_{\mathrm{s}}$ projects, we located the visual areas based on callosal connectivity as a landmark ${ }^{39-41}$; the callosal terminals expressing green fluorescent protein (GFP) visualized the boundaries among the primary visual cortex (V1) and higher visual areas in vivo (Fig. $4 \mathrm{a}-\mathrm{d}$ ). The $\mathrm{MO}_{\mathrm{s}}$ projections formed a prominent band of tdTomato fluorescence, the lateral part of which was located rostrolateral to the GFP-labeled boundaries between the V1 and higher visual areas. In particular, the band of $\mathrm{MO}_{\mathrm{s}}$ projections overlapped with a ring structure of callosal connectivity, which represented area RL (ref. ${ }^{39}$, also known as the 'rostrolateral visual area' in the nomenclature of the Allen Reference atlas ${ }^{7}$; Fig. 4c, d). In addition to area RL, the band appeared to coincide with areas A ('anterior area') and AL ('anterolateral') in the visual cortex and to a small degree with the V1. We confirmed this pattern of $\mathrm{MO}_{\mathrm{s}}$ projections with Nissl-stained coronal sections (Fig. 4j, m) where areas $\mathrm{RL}, \mathrm{A}$, and $\mathrm{AL}$ belong to a single cytoarchitectural region, the lateral area of the secondary visual cortex (V2L, the nomenclature of Paxinos and Franklin's atlas ${ }^{42}$ ). Again, the $\mathrm{MO}_{\mathrm{s}}$ projections coincided weakly with the V1 but well with V2L (Fig. $4 \mathrm{~m}$ ). These rostrolateral higher visual areas likely receive information related to eye movements from the $\mathrm{MO}_{\mathrm{s}}$, consistent with the hypothesis that the RL, A, and AL areas fit in an anatomical diagram that resembles the dorsal visual stream ${ }^{41}$ specialized for visuospatial processing and guiding actions ${ }^{43}$. However, unlike the $\mathrm{MO}_{\mathrm{s}}$, electrical stimulation in these areas rarely evoked eye movements (Supplementary Fig. 11) unless the stimulation current was doubled or tripled (up to over $200 \mu \mathrm{A}$ ), supporting the notion that these are mainly sensory areas. This study refers to these rostrolateral higher visual areas receiving $\mathrm{MO}_{\mathrm{s}}$ projections as the $\mathrm{V}_{\mathrm{RL} / \mathrm{A} / \mathrm{AL}}$.

A previous study reported projections from the $\mathrm{RL}, \mathrm{A}$, and $\mathrm{AL}$ areas to the secondary motor area ${ }^{41}$, suggesting that the longrange connections between the $\mathrm{MO}_{s}$ and the $\mathrm{V}_{\mathrm{RL} / \mathrm{A} / \mathrm{AL}}$ are reciprocal. To confirm the reciprocal connectivity, we conducted 

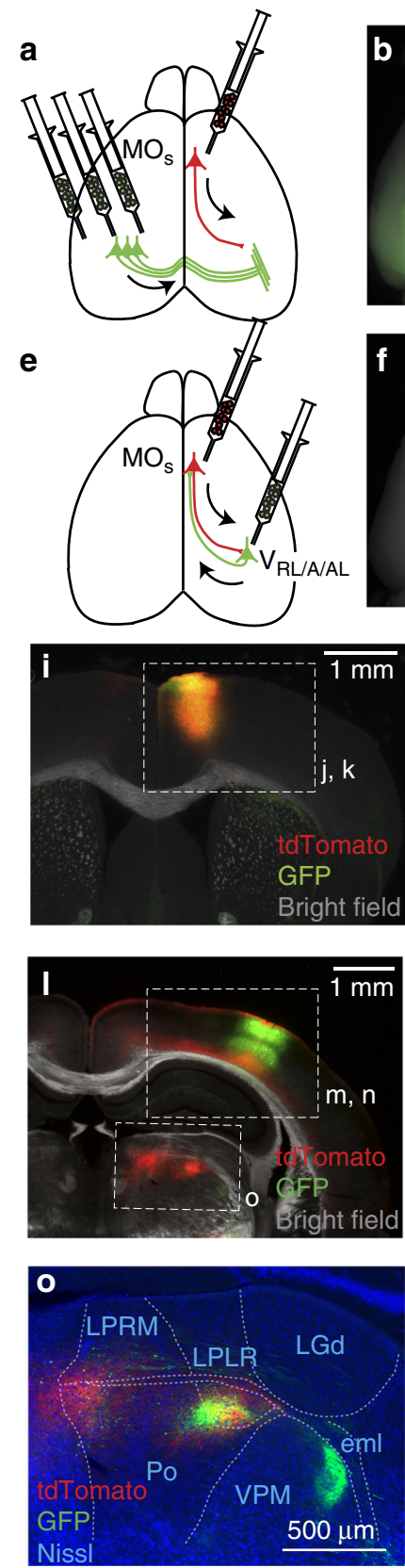
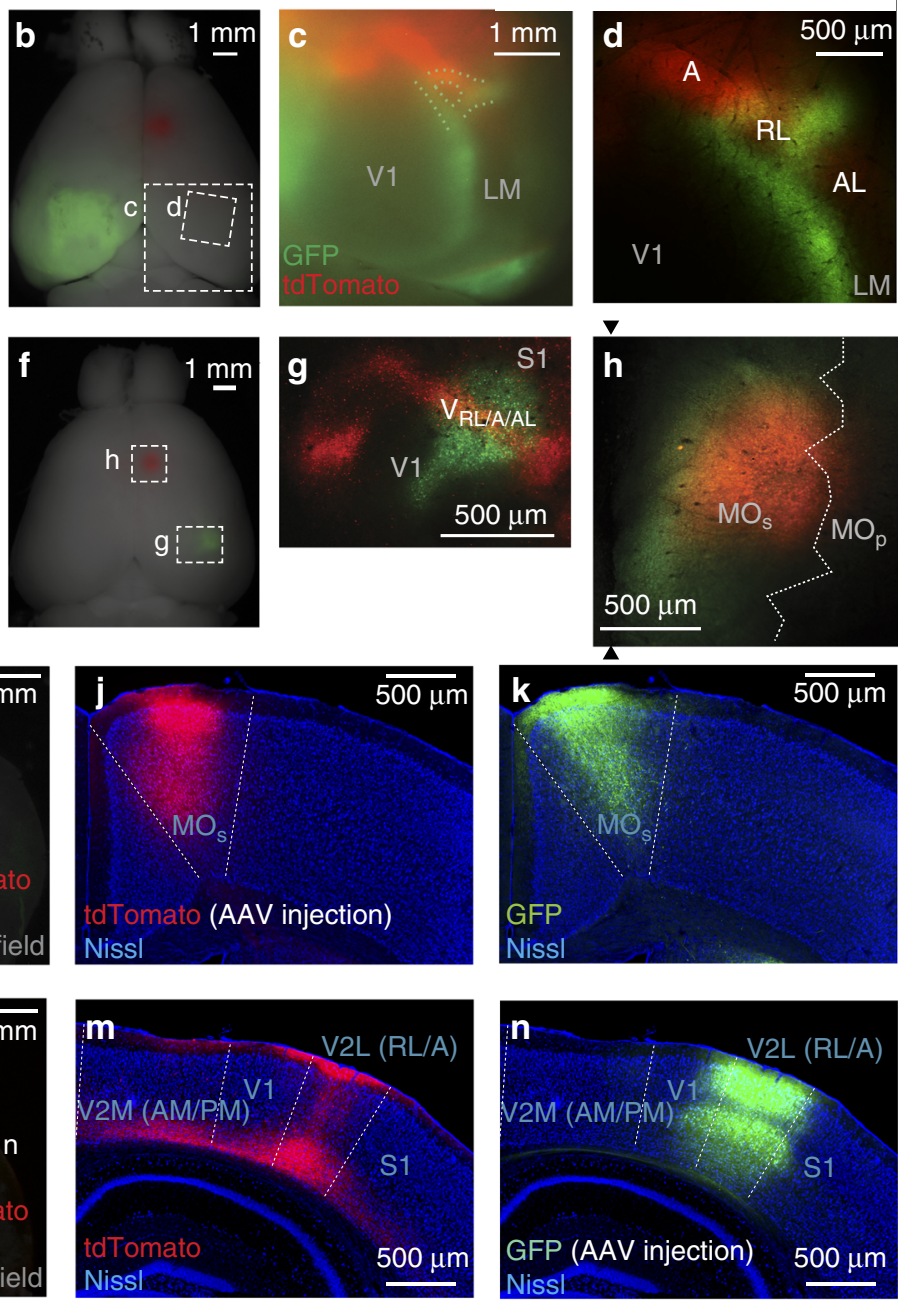

Fig. 4 Reciprocal connectivity between $M_{S}$ and $V_{R L / A} / A L$. a-d Connectivity from the $M O_{s}$ to the $V_{R L / A}$ AL. a The experimental design for the dual-virus injections. AAV-tdTomato was injected into the $\mathrm{MO}_{\mathrm{s}}$ in the right hemisphere and AAV-GFP was injected into the occipital regions in the left hemisphere. $\mathbf{b}$ A corresponding whole-brain image. c, d The tdTomato projection from $M_{S}$ overlapped with areas RL, $A$, and $A L$ ( $\left.V_{R L} / A / A L\right)$. GFP-labeled callosal projections delineated boundaries between the $\mathrm{V} 1$ and higher visual areas. The image $\mathbf{c}$ was taken with a fluorescent stereoscopic microscope from the dotted rectangle in $\mathbf{b}$, and the image $\mathbf{d}$ was taken with a two-photon microscope from a region indicated in $\mathbf{b}$. e-n The $\mathrm{V}_{\mathrm{RL} / \mathrm{A} / \mathrm{AL}} \mathrm{projects}$ back to the $\mathrm{MO}_{\mathrm{S}}$. $\mathbf{e}$ The experimental design for the sequential virus injections. First, AAV-tdTomato was injected into the MOs, and then a couple weeks later AAV-GFP was injected into the tdTomato-labeled axonal band (the $V_{R L / A} A L$ ). $\mathbf{f} A$ corresponding whole-brain image. $\mathbf{g}$ At the injection site of $A A V$ - GFP, the expression overlapped with the tdTomato-labeled axonal band (the location of $\mathrm{V}_{\mathrm{RL} / \mathrm{A} / \mathrm{AL}}$ ). $\mathbf{h}$ At the injection site of $A \mathrm{AV}$-tdTomato (the $\mathrm{MO}$ ), the expression overlapped with the GFP-labeled axonal band. The two black arrowheads indicate the midline, and the white dotted line is the expected areal border based

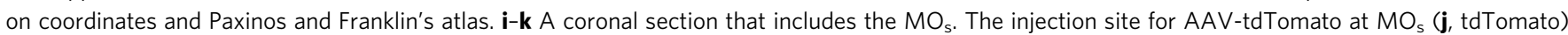

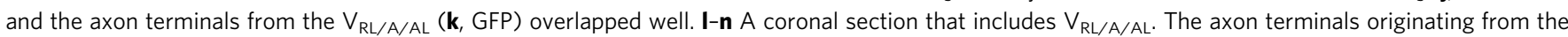
$\mathrm{MO}_{\mathrm{s}}$ ( $\mathbf{m}$, tdTomato) and the inection site for AAV-GFP (n, GFP) overlapped well. The dashed lines in $\mathbf{j}$, $\mathbf{k}, \mathbf{m}$, and $\mathbf{n}$ indicate the areal borders based on Nissl staining. o Projections from the $\mathrm{MO}_{\mathrm{s}}$ and the $\mathrm{V}_{\mathrm{RL} / \mathrm{A} / \mathrm{AL}}$ in thalamus. Note that projections from the $\mathrm{V}_{\mathrm{RL} / \mathrm{A} / \mathrm{AL}}$ to the higher visual thalamus (LPLR) 73 overlapped with those from the $\mathrm{MO}_{\mathrm{s}}$. The borders were drawn based on descriptions in refs. 42,74 
a

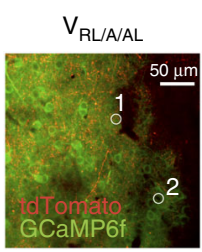

b

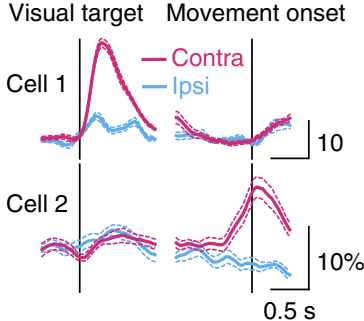

C

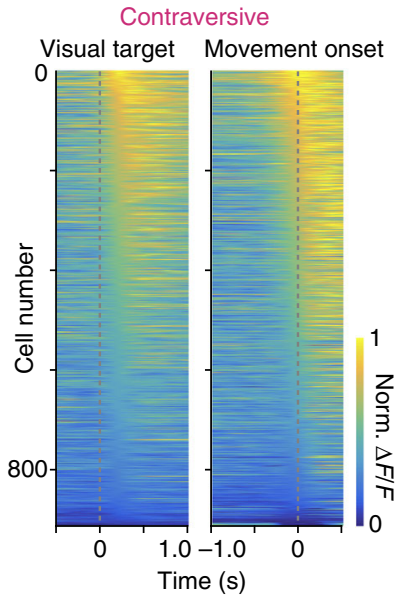

d

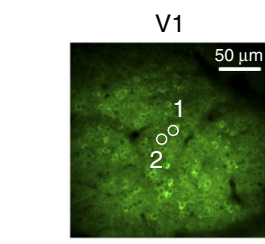

e

Visual target Movement onset

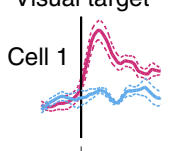

f

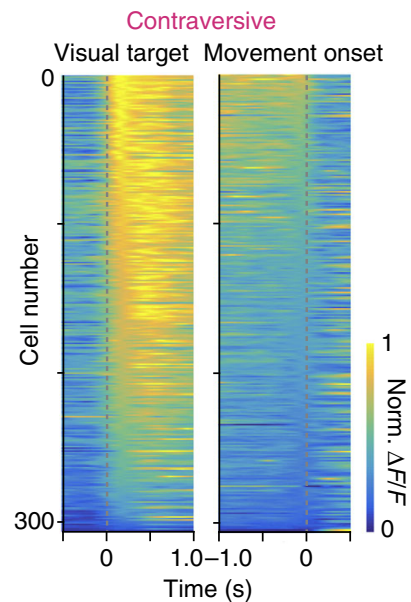

g

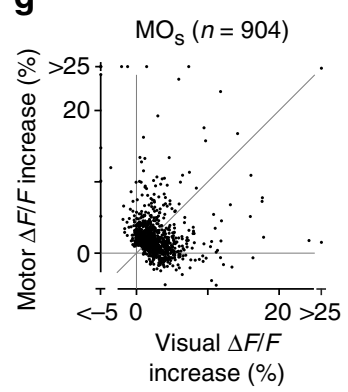

h

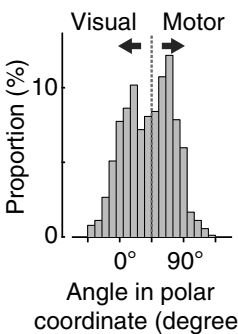

coordinate (degree) i

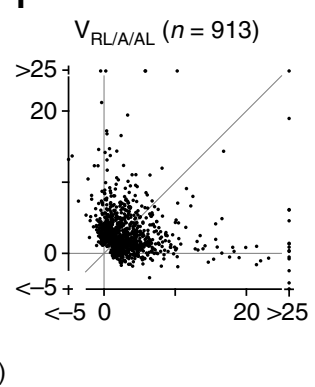

j

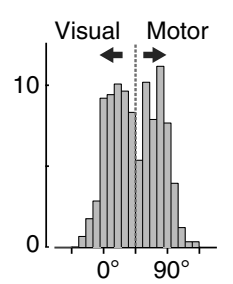

k

$\mathrm{V} 1(n=306)$

I
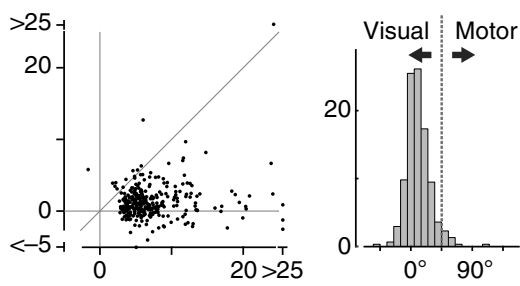

Fig. 5 Neural coding in the $\mathrm{V}_{\mathrm{RL} / \mathrm{A} / \mathrm{AL}}$ and the $\mathrm{V} 1$. a-c Coding in the $\mathrm{V}_{\mathrm{RL} / \mathrm{A} / \mathrm{AL}}$. a GCaMP6f expression at layer $2 / 3$ in the $\mathrm{V}_{\mathrm{RL} / \mathrm{A} / \mathrm{AL}}$. The position of the $\mathrm{V}_{\mathrm{RL} / \mathrm{A} /}$ AL was determined with fluorescent guidance of tdTomato-labeled projections from the $\mathrm{MO}_{\mathrm{s}}$. $\mathbf{b}$ Representative cells. Corresponding ROIs are shown in a. $\mathbf{C}$ Sorted normalized $\Delta F / F$ responses for neurons showing significant response $(n=913)$. Traces were aligned to either the onset of the visual target or the movement in the contraversive movement condition. d-f Coding in the V1. d GCaMP6f expression at layer 2/3 in the V1. e Representative cells. $\mathbf{f}$ Sorted normalized $\Delta F / F$ responses for neurons with a significant response $(n=306)$. $\mathbf{g}-\mathbf{I}$ Comparison between visual and motor activity for neurons in the $M \mathrm{O}_{\mathrm{s}}$ ( $\mathbf{g}, \mathbf{h}, n=904), \mathrm{V}_{\mathrm{RL} / \mathrm{A} / \mathrm{AL}}(\mathbf{i}, \mathbf{j} n=913)$, and $\mathrm{V} 1(\mathbf{k}, \mathbf{I}, n=306) . \mathbf{g}, \mathbf{i}, \mathbf{k}$ Visual and motor activity was plotted for each neuron. $\mathbf{h}, \mathbf{j}, \mathbf{I}$ The angle in polar coordinates was computed for each neuron and plotted as a histogram. Degrees of 0 or 90 indicate pure visual or motor activity

sequential virus injections (Fig. 4e). We delivered the first virus (AAV-tdTomato) to the $\mathrm{MO}_{\mathrm{s}}$ (Fig. 4f, $\mathrm{h}, \mathrm{j}$ ) to visualize its

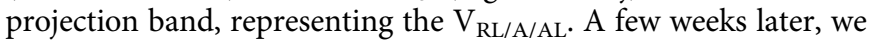
injected a second virus (AAV-GFP, green signal in Fig. $4 \mathrm{~g}, \mathrm{n}$ ) into the middle of the tdTomato band (red signal in Fig. $4 \mathrm{~g}, \mathrm{~m}$, expected to be around the RL) to label the projections from the $\mathrm{V}_{\mathrm{RL} / \mathrm{A} / \mathrm{AL}}$. Through this sequential approach, we found that GFPlabeled projections from the $\mathrm{V}_{\mathrm{RL} / \mathrm{A} / \mathrm{AL}}$ overlapped with the first injection site (AAV-tdTomato) in the $\mathrm{MO}_{\mathrm{s}}$ (Fig. 4h, i). Therefore, we conclude that the $\mathrm{MO}_{s}$ interacts with the $\mathrm{V}_{\mathrm{RL} / \mathrm{A} / \mathrm{AL}}$ through long-range reciprocal connectivity.

We further confirmed the strong reciprocal connections between the $\mathrm{MO}_{\mathrm{s}}$ and the $\mathrm{V}_{\mathrm{RL} / \mathrm{A} / \mathrm{AL}}$ with a database available from the Allen Institute for Brain Science ${ }^{7}$. Virus injected into coordinates $500-1000 \mathrm{~mm}$ rostral and lateral to the bregma (designated as the secondary motor area in their nomenclature) labeled a prominent axonal band similar to ours, which covered areas RL, A, and AL (Supplementary Fig. 12a-c). The axonal density in these areas was $14.0 \pm 4.2$ times higher than in the V1 ( $n=7$, Supplementary Fig. 12f). Again similar to our results, virus injection into area $\mathrm{RL} / \mathrm{A} / \mathrm{AL}$ labeled axon terminals densely at coordinates $\sim 700 \mathrm{~mm}$ rostral and lateral to the bregma in the secondary motor cortex (Supplementary Fig. 12h-j, m). Injection into the V1 labeled more selectively in the anterior cingulate area $^{44}$ (Supplementary Fig. 12n). The database supports that the $\mathrm{MO}_{\mathrm{s}}$ and the $\mathrm{V}_{\mathrm{RL} / \mathrm{A} / \mathrm{AL}}$ are strongly linked with each other. In addition, the database confirms the parallel reciprocal pathways reported in the frontal-parieto-occipital cortex ${ }^{44,45}$; the secondary motor area $\sim 0.5 \mathrm{~mm}$ rostrolateral to our injection site is reciprocally linked with the primary somatosensory cortex (Supplementary Fig. 12e, 1), and the dorsal part of the anterior cingulate area is linked with the V1 (Supplementary Fig. 12g, n). However, these three sets of reciprocal connections overlapped with each other, indicating that the connectivity was diffuse. Altogether, the database illustrates reciprocal connectivity in frontal-parieto-occipital cortex, including the $\mathrm{MO}_{\mathrm{s}}$ and the $\mathrm{V}_{\mathrm{RL} / \mathrm{A} / \mathrm{AL}}$.

Mixed encoding of visual/motor signal in $\mathrm{V}_{\mathrm{RL} / \mathrm{A} / \mathrm{AL}}$ but not in V1. The strong reciprocal connections between the $V_{R L / A / A L}$ and the $\mathrm{MO}_{\mathrm{s}}$ raised the possibility that neural activity in the $\mathrm{V}_{\mathrm{RL} / \mathrm{A} / \mathrm{AL}}$ is similar to that in the $\mathrm{MO}_{\mathrm{s}}$. Therefore, we investigated neural activity in the $\mathrm{V}_{\mathrm{RL} / \mathrm{A} / \mathrm{AL}}$ by measuring fluorescence changes of virally expressed GCaMP6f from layer $2 / 3$ neurons. Neurons in the $\mathrm{V}_{\mathrm{RL} / \mathrm{A} / \mathrm{AL}}$, which we identified by labeling the $\mathrm{MO}_{\mathrm{s}}$ projection terminals with tdTomato (Fig. 5a, red signal, Supplementary Fig. 13a), showed a visual response and motor activity, similar to the $\mathrm{MO}_{\mathrm{s}}$ (visual activity, $23 \%$ of 2321 neurons; motor activity, $20 \%$, Fig. $5 \mathrm{~b}, \mathrm{c}$ ). The $\mathrm{V}_{\mathrm{RL} / \mathrm{A} / \mathrm{AL}}$ also had more neurons that preferred contraversive movement (Supplementary Fig. 14c, d). In contrast, the $\mathrm{V} 1$, which was only weakly connected with the $\mathrm{MO}_{s}$, showed little motor activity (visual activity, 57\% of 531 neurons; motor activity, $0.9 \%$ of 531 neurons; Fig. $5 \mathrm{~d}-\mathrm{f}$ ). To evaluate the preference for visual or motor activity in the $\mathrm{V} 1, \mathrm{MO}_{\mathrm{s}}$, and $\mathrm{V}_{\mathrm{RL} / \mathrm{A} / \mathrm{AL}}$, we computed the polar angle for individual neurons 
a
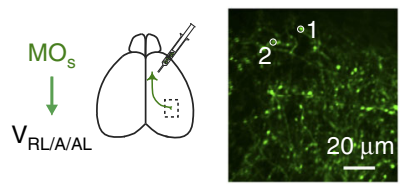

b Visual Axon $1 \mid \begin{aligned} & \text { - Contra } \\ & \text { - Ipsi }\end{aligned}$ Movement
onset

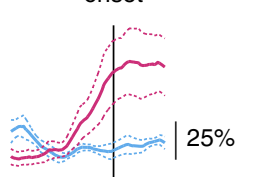

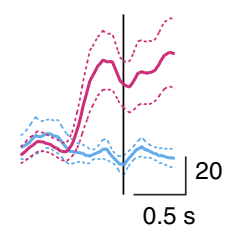

i

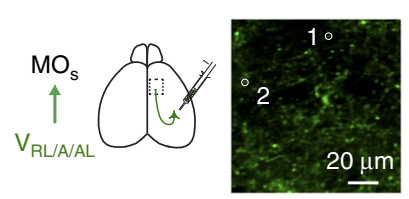

j
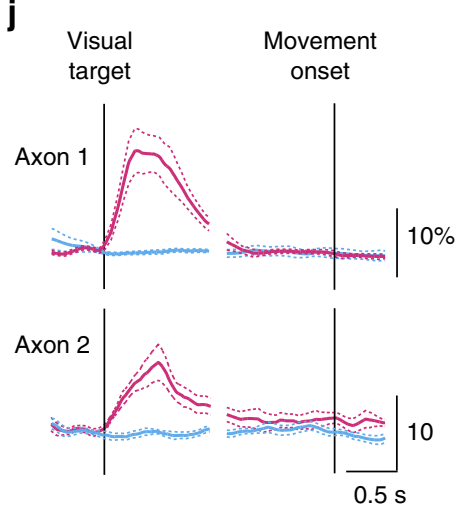

C

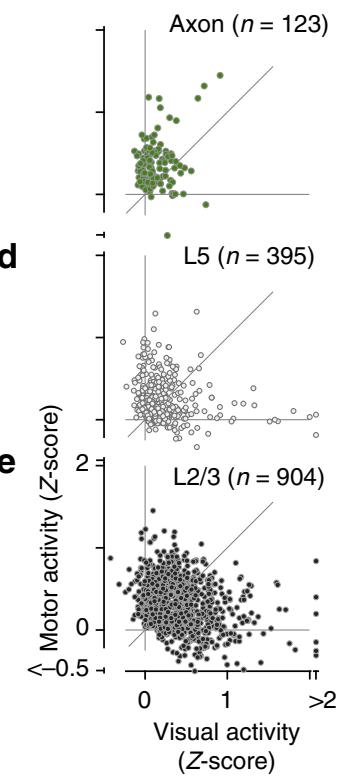

k

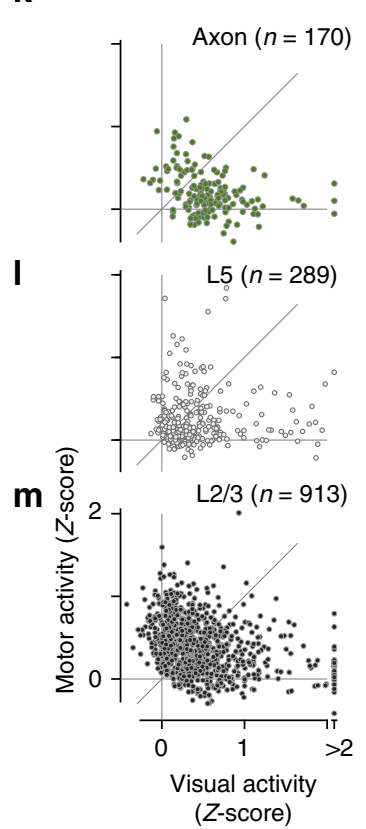

f

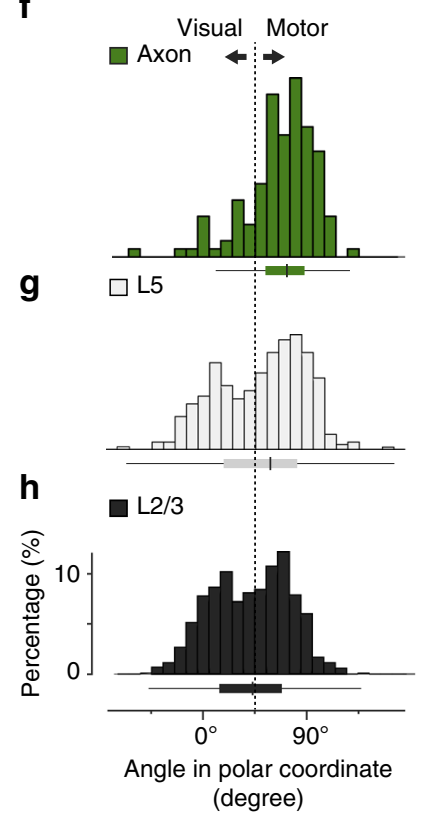

n
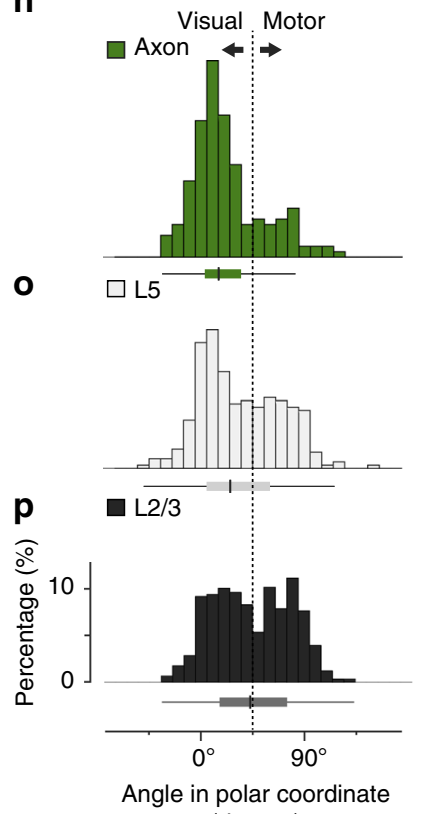

(degree)

Fig. 6 Biased neural coding in $M_{s}$ and $V_{R L / A / A L}$ axons. a-c Coding in $M_{S}$ axon terminals projecting to the $V_{R L / A / A L}$. a GCaMP6f was expressed in $M O_{s}$

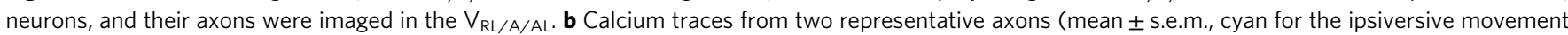
condition; magenta for the contraversive). The corresponding ROls are shown in a. c-e Comparison between visual and motor activity for $\mathrm{MO}_{\mathrm{s}}$ axons (c, $n=123$ ), L5 somas $(\mathbf{d}, n=395)$, and L2/3 somas $(\mathbf{e}, n=904)$. $\mathbf{f}-\mathbf{h}$ Histograms of the angle in polar coordinates for $\mathrm{MO}_{\mathrm{s}}$ axons, $\mathrm{L} 5 \mathrm{meurons}$, and $\mathrm{L} 2 / 3$ neurons. The distribution of the axons is more biased towards 90 degrees, indicating higher motor selectivity. Median, 25\% quantile, and $75 \%$ quantile are represented as box plots, whisker length is 1.5 of the interquartile range. i-p Similar to $\mathbf{a}-\mathbf{h}$, but for $\mathrm{V}_{\mathrm{RL} / \mathrm{A} / \mathrm{AL}}$ axon terminals projecting to the $\mathrm{MO}_{\mathrm{s}}$. $\mathrm{V}_{\mathrm{RL} / \mathrm{A} / \mathrm{AL}}$ axons, $n=170 ; V_{R L / A}$ AL $L 5$ somas, $n=289 ; L 2 / 3$ somas, $n=913$. Note skewed distribution of the axons towards 0 degrees, indicating higher visual selectivity. The histograms for $L 2 / 3$ somas $(\mathbf{h}, \mathbf{p})$ are the same with those in Fig. $5 \mathrm{~h}, \mathrm{j}$

based on the Cartesian representation of visual and motor activity (Fig. $5 \mathrm{~g}, \mathrm{i}, \mathrm{k}$ ). In the polar representation, the angle of $90^{\circ}$ indicates strong preference for motor information, and $0^{\circ}$ for visual information. The distribution of the polar angles indicate that the preference for motor over visual activity across neurons in the $\mathrm{V}_{\mathrm{RL} / \mathrm{A} / \mathrm{AL}}$ was significantly different from the V1 but not the $\mathrm{MO}_{\mathrm{s}}$ (Fig. 5h, j, l, $\mathrm{MO}_{\mathrm{s}}, n=904, \mathrm{~V}_{\mathrm{RL} / \mathrm{A} / \mathrm{AL}}, n=913, \mathrm{~V} 1, n=306$; $p<10^{-43}$ and $p=0.06$, respectively, Mann-Whitney test). Preference for motor activity over post-movement activity was similarly evaluated and was found to be slightly less in the $\mathrm{V}_{\mathrm{RL} / \mathrm{A} /}$ AL than the $\mathrm{MO}_{\mathrm{s}}$ (Supplementary Fig. 15b, d, MO,$n=669$, $\mathrm{V}_{\mathrm{RL} / \mathrm{A} / \mathrm{AL}}, \quad n=705 ; p<0.001$, Mann-Whitney test) but much stronger than the V1 (Supplementary Fig. 15d, f, $\mathrm{V}_{\mathrm{RL} / \mathrm{A} / \mathrm{AL}}$, $n=705$; V1, $n=54 ; p<10^{-9}$, Mann-Whitney test, the postmovement activity represents an intricate mixture of visual activity, remaining motor signals, and post-movement motorrelated activity $\left.{ }^{36}\right)$. These results indicate that the $\mathrm{MO}_{\mathrm{s}}$ and the $\mathrm{V}_{\mathrm{RL} / \mathrm{A} / \mathrm{AL}}$ encode qualitatively similar patterns of visual and motor 
activity and suggest that the reciprocally connected sensory and motor areas form a distributed network in sensory-motor processing.

Direction-dependent flow of sensory-motor information. The similar encoding patterns in the $\mathrm{MO}_{\mathrm{s}}$ and the $\mathrm{V}_{\mathrm{RL} / \mathrm{A} / \mathrm{AL}}$ raise the question of how these two areas communicate with each other in a distributed network. One possibility is that the connections between these two areas underlie reverberant neural activity; the reciprocal connections might carry similar information in both directions (Supplementary Fig. 1b). Alternatively, the connections between them might preferentially convey specific types of information depending on the direction of the connectivity, indicating streamlined information flow in the distributed network (Supplementary Fig. 1c). To distinguish between these possibilities, we imaged calcium signals of projected axon terminals ${ }^{18}$ in two directions: from the $\mathrm{MO}_{s}$ to the $\mathrm{V}_{\mathrm{RL} / \mathrm{A} / \mathrm{AL}}$ (Fig. 6a, b) and from the $\mathrm{V}_{\mathrm{RL} / \mathrm{A} / \mathrm{AL}}$ to the $\mathrm{MO}_{\mathrm{s}}$ (Fig. $6 \mathrm{i}$, j, Supplementary Fig. 13b). Compared to layer $2 / 3$ neurons in the $\mathrm{MO}_{\mathrm{s}}$, axon terminals from the $\mathrm{MO}_{\mathrm{s}}$ to the $\mathrm{V}_{\mathrm{RL} / \mathrm{A} / \mathrm{AL}}$ showed a polar angle more distributed around $90^{\circ}$ (Fig. $6 \mathrm{c}, \mathrm{f}$ ), exhibiting more motor activity than visual activity $\left(p<10^{-14}\right.$, Mann-Whitney test, 123 axon terminals vs. 904 layer $2 / 3$ somas, Fig. $6 f, h)$. Higher selectivity for motor information in axon terminals indicates that the $\mathrm{MO}_{\mathrm{s}}$ favorably conveys motor signals to the $\mathrm{V}_{\mathrm{RL} / \mathrm{A} / \mathrm{AL}}$. In contrast, axon terminals from the $\mathrm{V}_{\mathrm{RL} / \mathrm{A} / \mathrm{AL}}$ to the $\mathrm{MO}_{\mathrm{s}}$ (Fig. $6 \mathrm{k}$, $\mathrm{n})$ showed a stronger preference for visual activity compared to layer $2 / 3$ neurons in the $\mathrm{V}_{\mathrm{RL} / \mathrm{A} / \mathrm{AL}}\left(p<10^{-13}, 170\right.$ axon terminals vs. 913 layer $2 / 3$ somas, Fig. $6 n, p)$. In addition to layer $2 / 3$ neurons, another major source of long-range axonal projections is layer 5a neurons, which showed higher selectivity than layer $2 / 3$ neurons in the $\mathrm{MO}_{\mathrm{s}}\left(p<10^{-4}\right.$, Mann-Whitney test, 395 layer 5a somas: Fig. 6d, g, 904 layer 2/3 somas: Fig. 6e, h) and in the $\mathrm{V}_{\mathrm{RL} / \mathrm{A} /}$ AL $\left(p<10^{-5}\right.$, Mann-Whitney test, 289 layer 5a somas: Fig. 61, o, 913 layer 2/3 somas: Fig. $6 \mathrm{~m}, \mathrm{p})$. Nonetheless, we observed even higher selectivity in axon terminals than in layer 5 a neurons in both the $\mathrm{MO}_{\mathrm{s}}\left(p<10^{-4}\right.$, Mann-Whitney test, 123 axon terminals vs. 395 layer 5a somas; Fig. $6 \mathrm{f}, \mathrm{g})$ and the $\mathrm{V}_{\mathrm{RL} / \mathrm{A} / \mathrm{AL}}\left(p<10^{-3}\right.$, Mann-Whitney test, 170 axon terminals vs. 289 layer 5a somas; Fig. 6n, o). This direction-dependent information flow between the $\mathrm{MO}_{\mathrm{s}}$ and the $\mathrm{V}_{\mathrm{RL} / \mathrm{A} / \mathrm{AL}}$ suggests that the reciprocal connectivity acts as a bidirectional filter in the distributed network.

\section{Discussion}

The cerebral cortex consists of intricate networks of long-range connections, many of which are reciprocal. Such reciprocal connectivity is particularly prominent between sensory and motor cortical areas, and yet how the networks operate in vivo through the connectivity has been little investigated. In this study, we discovered that communication through reciprocal connections between the $\mathrm{MO}_{\mathrm{s}}$ and the $\mathrm{V}_{\mathrm{RL} / \mathrm{A} / \mathrm{AL}}$ depends on the direction of the connectivity, suggesting that they function as a bidirectional filter. Thus our findings provide insight into how reciprocal long-range connectivity contributes to communication within a distributed network of cortical areas.

Our study showed that mice possess basic cortical circuits for eye movement, in addition to the circuits for the visual system that have been investigated intensively ${ }^{46}$. Although eye movements have been reported in awake rodents head-fixed at rest $^{47-49}$ or running ${ }^{50}$ and in those freely moving ${ }^{51}$, it was unclear whether those movements were produced voluntarily. Our approach, which incorporated an eye movement task, optogenetic suppression, and in vivo two-photon imaging, provided converging evidence that mice are capable of shifting their eyes voluntarily. Furthermore, we found that these binocularly coupled eye movements were controlled by a small portion of the frontal cortex, located within the secondary motor cortex. It was unexpected that this area controls binocularly coupled eye movements, as mice have afoveate retinas, a limited binocular visual field due to their lateralized eyes, and independent control over their two eyes by vestibular inputs ${ }^{51}$. One possible explanation for these binocularly coupled eye movements is that they might play a role in maintaining binocular visual fusion. Although the binocular coupled eye movements in mice were qualitatively similar to those in primates, and the projection patterns of the mouse $\mathrm{MO}_{\mathrm{s}}$ to higher visual areas were reminiscent of those of the primate frontal eye field $(\mathrm{FEF})^{52}$, variable amplitude of eye movements at the same electrical stimulation current (Supplementary Fig. 5a), together with the lack of distinct cytoarchitecture ${ }^{42}$, contrasts with the primate FEF. Therefore, it is premature to conclude that this portion of the secondary motor cortex is specialized for eye movements or is a primitive homolog of primate cortical areas for eye movements (i.e., the FEF (ref. ${ }^{33}$ ), the supplemental eye field $(\mathrm{SEF})^{35)}$, and the rat frontal orienting field $(\mathrm{FOF})^{53}$. Future studies will be needed to determine whether this $\mathrm{MO}_{\mathrm{s}}$ portion contains the same highly sophisticated functions and anatomical connectivity as the primate FEF, SEF, and the rat FOF.

Our visually guided eye movement task provided us with a unique opportunity to investigate how motor activity is represented in the visual cortex, which consists of the V1 and higher visual areas. These visual areas are known to have their own preferences for particular visual stimuli ${ }^{5,6}$ and our finding also shows their distinct preferences for eye motor signals, prominent in higher areas of the dorsal stream ${ }^{41}$ (i.e., the $\mathrm{V}_{\mathrm{RL} / \mathrm{A} / \mathrm{AL}}$ ) but little in the V1. Similarly, the $\mathrm{V}_{\mathrm{RL} / \mathrm{A} / \mathrm{AL}}$ showed a stronger preference for eye motor signals over post-movement activity than the V1, although this post-movement activity is not straightforward to interpret because of the intricate mixture of visual activity, remaining motor signal, and post-movement motor-related activity ${ }^{36}$. These distinct preferences for eye motor signals coincided with anatomical projections from the $\mathrm{MO}_{\mathrm{s}}$ to the visual cortex; the $\mathrm{MO}_{\mathrm{s}}$ sent strong projections not to the V1 but to the $\mathrm{V}_{\mathrm{RL} / \mathrm{A} / \mathrm{AL}}$. Therefore, motor signals in visual areas might be shaped by projections from the $\mathrm{MO}_{\mathrm{s}}$, which is more biased to motor information.

We found that the transfer of neural information between the $\mathrm{MO}_{\mathrm{s}}$ and the $\mathrm{V}_{\mathrm{RL} / \mathrm{A} / \mathrm{AL}}$ depended on the direction of the longrange connections. Previous studies have investigated how longrange connectivity carries information in the outgoing directions: how the primary somatosensory area sorts and conveys specific information to the secondary somatosensory area and to the motor cortex ${ }^{11-13}$. However, whether and how long-range connections convey specific information in different directions in reciprocal connectivity was unclear. A recent study suggested the intriguing possibility that the reciprocal connectivity between the primary somatosensory area and the secondary motor area serves as a recurrent loop to generate reverberant activity ${ }^{54}$. Our study reveals another possibility that the reciprocal connectivity in the visuo-oculomotor cortical circuit involving the $\mathrm{V}_{\mathrm{RL} / \mathrm{A} / \mathrm{AL}}$ preferentially conveys different types of information depending on the direction of information flow. Future research will be required to understand the physiological role of this reciprocal connectivity. For instance, it would be interesting to determine whether the selected information is used in the motor area for visuomotor transformation ${ }^{55}$ and in the visual areas for remapping of visual space and visuospatial attention ${ }^{56}$ as in primates.

What are the computational advantages to streamlining the information flow between the reciprocally connected areas of mixed sensory and motor representations? Streamlined flow might indicate the existence of processing units that are functionally localized even in a distributed network. Another 
possibility is that streamlined inflow in a distributed network may drive mixed selectivity; streamlined inflow from separate input sources might easily achieve mixed selectivity, which is known to be computationally powerful ${ }^{57-59}$. It will be interesting to investigate which of these possibilities, streamlined flow or mixed selectivity, is more strongly linked to behavioral performance ${ }^{57}$. Our findings may provide a foundation for investigating the computational power of reciprocal connectivity, which is abundant in the cortex.

\section{Methods}

Mice. All experimental procedures were approved by the University of Tübingen, National Center of Neurology and Psychiatry, and local institutions in charge of animal experiments. C57BL/6J and PV-Cre mice (JAX stock $\# 008069)^{60}$ were used in this study, including 10 mice for binocular eye movement analysis, 11 for microstimulation, 17 for optogenetic inhibition, 9 for anatomical tracing, and 35 for two-photon imaging ( 8 for imaging of $\mathrm{MO}_{\mathrm{s}}$ layer $2 / 3,6$ for $\mathrm{V}_{\mathrm{RL} / \mathrm{A} / \mathrm{AL}}$ layer $2 / 3,2$ for $\mathrm{V} 1$, seven for $\mathrm{MO}_{\mathrm{s}}$ layer 5, 4 for $\mathrm{V}_{\mathrm{RL} / \mathrm{A} / \mathrm{AL}}$ layer 5, 5 for axon terminals from $\mathrm{MO}_{\mathrm{s}}$, and 3 for axon terminals from $\mathrm{V}_{\mathrm{RL} / \mathrm{A} / \mathrm{AL}}$ ). All wild-type mice were randomly assigned to the experiments. For the optogenetic suppression experiment, Crepositive mice were selected by genotyping and then assigned randomly to each of the groups. All mice were male and aged $>8$ weeks. The mice were group housed (up to five mice in a cage), and experiments were performed during the dark period of the $12 \mathrm{~h} \mathrm{light} / 12 \mathrm{~h}$ dark cycle.

All surgical procedures were carried out aseptically under anesthesia with 100 $\mathrm{mg} / \mathrm{kg}$ ketamine and $8 \mathrm{mg} / \mathrm{kg}$ xylazine (intraperitoneally), supplemented with isoflurane for maintenance. Lidocaine (subcutaneously at the incision), atropine $(0.3 \mathrm{mg} / \mathrm{kg}$, intraperitoneally), caprofen $(5 \mathrm{mg} / \mathrm{kg}$, intraperitoneally), and dexamethasone $(2 \mathrm{mg} / \mathrm{kg}$, intraperitoneally) were applied to prevent pain and brain edema. After surgery, mice were allowed to recover for at least 3 days. No experimenter blinding was done.

Cranial window surgery. A custom-made headpost was glued and cemented to the skull, and then a craniotomy (1-2 mm circle or rectangle) was performed over the $\mathrm{MO}_{\mathrm{s}}$ (Fig. $3 \mathrm{~b}, \sim 0.7 \mathrm{~mm}$ anterior and $\sim 0.7 \mathrm{~mm}$ lateral from the bregma, which is the best-fit intersection between the midline and the coronal suture), the $\mathrm{V}_{\mathrm{BL} / \mathrm{A} / \mathrm{AL}}(2.0$ $\mathrm{mm}$ posterior and $2.5 \mathrm{~mm}$ lateral from the bregma), or the $\mathrm{V} 1(0.0 \mathrm{~mm}$ posterior and $3.0 \mathrm{~mm}$ lateral from the lambda, which is the best-fit intersection between the midline and the lambdoid suture). The location of the $\mathrm{V}_{\mathrm{RL} / \mathrm{A} / \mathrm{AL}}$ was guided by the axon terminals of the $\mathrm{MO}_{\mathrm{s}}$ (see below). Inside the area of the craniotomy (above the $\mathrm{MO}_{s}, \mathrm{~V}_{\mathrm{RL} / \mathrm{A} / \mathrm{AL}}$, or V1), virus (AAV2/1-syn-GCaMP6f ${ }^{32}$ was injected at a couple of sites (40-60 $\mathrm{nl} / \mathrm{site}$; depth, $200-300 \mu \mathrm{m} ; 3-5 \mathrm{~min} / \mathrm{injection}$ ), which resulted in virus expression of $\sim 400 \mu \mathrm{m}$. An imaging window consisting of two or three layers of coverglass (a small trimmed glass piece glued onto a larger piece) was fixed to the skull with dental acrylic ${ }^{61}$ (Fig. 3b).

For axon terminal imaging, virus injection and imaging were performed in two different cortical areas (the $\mathrm{MO}_{\mathrm{s}}$ and $\left.\mathrm{V}_{\mathrm{RL} / \mathrm{A} / \mathrm{AL}}\right)$. A small hole $(250 \mu \mathrm{m})$ was made in the bone over the $\mathrm{MO}_{\mathrm{s}}$ (based on the coordinates) or $\mathrm{V}_{\mathrm{RL} / \mathrm{A} / \mathrm{AL}}$ (with the guide of callosal connectivity, see below). Through this hole, virus (AAV2/1-syn-GCaMP6f) 32 was injected at a depth of $200-300 \mu \mathrm{m}$. After virus injection, a headpost was implanted and a larger craniotomy $(1-2 \mathrm{~mm}$, circle or rectangle) was made above the other area (the $\mathrm{V}_{\mathrm{RL} / \mathrm{A} / \mathrm{AL}}$ or $\mathrm{MO}_{\mathrm{s}}$ ). Following craniotomy, a cranial glass window was implanted as described above. After virus injection, mice were allowed to recover in their home cages for a few days.

The position of the $\mathrm{V}_{\mathrm{RL} / \mathrm{A} / \mathrm{AL}}$ for GCaMP6f virus injection was identified based on axonal projections from the $\mathrm{MO}_{\mathrm{s}}$ or from the contralateral visual cortex (Supplementary Fig. 13). For somatic GCaMP6f imaging in the $\mathrm{V}_{\mathrm{RL} / \mathrm{A} / \mathrm{AL}}$, the injection location was determined with the guide of axon terminals from the $\mathrm{MO}_{\mathrm{s}}$ labeled beforehand with AAV2/1-EF1 $\alpha$-tdTomato or dextran conjugated with Alexa $594(10 \%$ v/w in cortex buffer, total of $400 \mathrm{nl}$, Supplementary Fig. 13a). The $M_{s}$ projections label areas RL and A heavily, thereby our injections were mainly in these areas. For imaging axon terminals from the $\mathrm{V}_{\mathrm{RL} / \mathrm{A} / \mathrm{AL}}$ to the $\mathrm{MO}_{\mathrm{s}}$, GCaMP6f virus was injected into area RL and A with a guide of the callosal ring visualized by injecting AAV2/1-EF1 $\alpha$-tdTomato or dextran Alexa 594 into the contralataral visual cortex beforehand (Supplementary Fig. 13b).

Behavioral training. Mice were pretrained to enter a tube to obtain a water reward (for 7-10 days). Over the last 2 days of pretraining, mice were acclimated to the imaging setup, and rapid eye movements were reinforced with a water reward. This reinforcement was crucial, as spontaneous eye movements became rare once the mice became used to the task environment. Following this pretraining, the mice were trained to perform a visually guided eye movement task. In this behavioral paradigm, three blue LEDs were used as visual stimuli: a central fixation LED, a nasal target LED, and a temporal target LED (wavelength $470 \mathrm{~nm}, \mathrm{M} 470 \mathrm{~F} 1$,

Thorlabs) (Supplementary Fig. 2). The fixation LED was placed $12 \mathrm{~cm}$ away from the left eye at an elevation angle of $20^{\circ}$ and an azimuth angle of $60^{\circ}$. The nasal and temporal target LEDs were placed at an elevation angle of $20^{\circ}$ and an azimuth angle of $35^{\circ}$ and $85^{\circ}$, respectively. In front of each LED, a 475-nm short-pass filter (\#84-692, OD4, Edmund) was placed to prevent stray light into the photomultiplier tube. A dichroic mirror (\#43-958, Edmund) was placed between the LED and the eye, allowing light below $675 \mathrm{~nm}$ to be transmitted and causing light above $750 \mathrm{~nm}$ to be reflected (Supplementary Fig. 2a). This strategy enabled infrared (IR) light to monitor eye position without interference from the fixation or target LEDs. During each trial, the brightness of the LEDs indicated where the left eye should be located (Fig. 1b). The brightness was measured in $\mu \mathrm{W}$ with a power meter (OP-2 VIS, Coherent) at the output of the LED. The light of the fixation LED was first set at $260 \mu \mathrm{W}$ for $3-4 \mathrm{~s}$ and then increased to $470 \mu \mathrm{W}$ at the start of the trial. When the left eye of the mouse was directed to the fixation LED (within \pm $2.5^{\circ}$ ), the brightness was further increased to $500 \mu \mathrm{W}$. The mouse was required to maintain the fixation for $750-1000 \mathrm{~ms}$. If the mouse failed to do so, the fixation LED became dimmer $(260 \mu \mathrm{W})$ for $1000 \mathrm{~ms}$ and then brighter again to indicate the beginning of the fixation period. The fixation break prior to the visual target onset was not considered an error. After successful fixation of 750-1000 ms, one of the two target LEDs (randomized) was turned on $(450 \mu \mathrm{W})$, and at the same time, the brightness of the fixation LED was decreased $(260 \mu \mathrm{W})$. The mouse was required to shift its gaze in the direction of the target LED within $10 \mathrm{~s}$, which was sufficiently long to keep its motivation. Following successful eye movements, the target LED was turned off ( $70 \mathrm{~ms}$ later), and after a short delay of $200-300 \mathrm{~ms}$, a drop of water was provided. The eye shift was required to be a rapid movement (amplitude $>5^{\circ}$, speed $>0.1 \% \mathrm{~ms}$ ). If the gaze moved out from the fixation window without the correct eye movement, the trial was considered as an error, an incorrect eye movement that can include a rapid movement in the wrong direction, a rapid movement at small amplitude, and a slow eye drift. The mouse generally performed this task $\sim 50$ times per day. For the imaging experiments, we encouraged mice to have longer reaction time by using a larger amount of reward for those trials (8-12 $\mu \mathrm{l}$ for reaction time shorter than $1 \mathrm{~s}, 20-25 \mu \mathrm{l}$ for reaction time longer than $1 \mathrm{~s}$ up to $20 \mathrm{~s}$ ). After doing this, the mice had a median reaction time of over $1 \mathrm{~s}$ (Supplementary Fig. 8); trials shorter than $1.5 \mathrm{~s}$ were excluded to facilitate separation of visual and motor activity.

The training procedure for this task was similar to that used for saccade tasks in primates ${ }^{62,63}$. Over the first several days, fixation to the central LED was encouraged with a small amount of water, and the task was made easier by having a large fixation window $\left( \pm 5^{\circ}\right)$. Upon successful eye movement, a larger reward (up to $50 \mu \mathrm{l}$ ) was provided. As the task performance improved, the reward amount was reduced $(15-25 \mu \mathrm{l})$ and the size of the fixation window was decreased to $\pm 2.5^{\circ}$, which rejects large eye drift and small eye movements. Some mice that stuck to eye drifts were excluded ( $n=10$ mice), as such drifts severely impeded improvement in behavioral performance. Behavior was monitored using a program written in TEMPO $^{62,63}$. When we instructed the mice to move their eyes vertically, we could not keep them motivated as a consequence of too many consecutive errors. This was also the case when we turned off the target LED before the onset of eye movement (a memory-guided eye movement task).

Measurement and analysis of eye position. The position of the left eye was monitored using a commercial camera package (Eyelink 1000, SR Research) sampled at $500 \mathrm{~Hz}$ during the task. The light source for the camera was a $940-\mathrm{nm}$ LED. In front of the camera, an 800-nm long pass filter (\#66-059, Edmund) and a 960-nm short pass filter (HQ960SP, Chroma) were placed to block the IR light for the right eye, blue LEDs, and two-photon laser. In some experiments, the image of the left eye was saved at $200 \mathrm{~Hz}$ with precise timing of individual frames, using a complementary metal-oxide semiconductor (CMOS) camera (DCC1240M, Thorlabs) that was controlled by custom-made software (written in Visual C++ with the Thorlabs Software Development Kit). The camera was placed at the same azimuth angle as the fixation LED. At the edge of the camera were four reference LEDs to confirm that the eye was directed toward the fixation $\mathrm{LED}^{47}$. Optical filters were similarly placed in front of the camera.

In some experiments, the position of the right eye was monitored using a second CMOS camera (DCC1240M, Thorlabs) with images saved at $200 \mathrm{~Hz}$. The light source for this camera was a $780 \mathrm{~nm}$ LED (M780L2, Thorlabs). In front of the camera, a 960-nm short pass filter (HQ960SP, Chroma) was placed.

The position of the eye was determined for each frame using a custom-written program in MATLAB (Supplementary Fig. 2b). The image in each frame (Supplementary Fig. 2b1) was processed to produce two binary maps: (1) a threshold map based on discrimination analysis (Supplementary Fig. 2b2), identifying the light source and eyelids; and (2) a Canny map based on the Canny edge detector ${ }^{64}$ (Supplementary Fig. 2b3), detecting the boundary of the pupil, light source, and eyelids. By combining these two maps, the edges corresponding to the light source and those within the eyelids could be eliminated (Supplementary Fig. 2b4, a candidate map). The boundary of the pupil was determined by searching the best-fit circle with a circumference overlapping as many white pixels per length as possible in the candidate map. Once the pupil boundary was identified, the angular position of the eye was calculated using $x, y$, and $r^{47}$.

Microstimulation. Electrical microstimulation was conducted in awake mice to identify a candidate cortical region for eye movements in the frontal cortex (Supplementary Fig. 5). The mouse was head-fixed in the behavioral apparatus, and the positions of the two eyes were monitored. When the left eye was in its primary 
position (azimuth angle of $\sim 60^{\circ}$ ), the stimulation was delivered using a tungsten or platinum/iridium electrode (amplitude, 20-50 $\mu \mathrm{A}$; frequency, $200 \mathrm{~Hz}$; pulse width, $300 \mu$ s; duration, $250 \mathrm{~ms}$ ) at around layer 5, at a depth of $700 \mu \mathrm{m}$ (or 1000-1500 $\mu \mathrm{m}$ for medial bank, region II in Supplementary Fig. $5 \mathrm{c}-\mathrm{e}$ ), and at $0.3-1.5 \mathrm{~mm}$ lateral to the bregma (or $0.4-0.7 \mathrm{~mm}$ for medial bank). The stimulation parameters were similar to those used in previous studies on motor regions in rodents ${ }^{65}$ and primates $^{34}$. The effects of microstimulation were also examined at the location of the $\mathrm{V}_{\mathrm{RL} / \mathrm{A} / \mathrm{AL}}$ and V1 (Supplementary Fig. 11). In most animals, the current value was fixed at the lowest value that could evoke reliable eye movements at $0.7 \mathrm{~mm}$ rostral and $0.7 \mathrm{~mm}$ lateral to the bregma, allowing many sites to be stimulated while the mice remained still. In two animals, we confirmed that a stronger current $(\sim 200$ $\mu \mathrm{A}$ ) could evoke eye movements in the anterior cingulate area, the $\mathrm{V}_{\mathrm{RL} / \mathrm{A} / \mathrm{AL}}$, and superficial layers (at $300 \mu \mathrm{m}$ ) of the $\mathrm{MO}_{\mathrm{s}}$.

In three experiments, we confirmed the electrode track in the $\mathrm{MO}_{\mathrm{s}}$ with coronal sections. The electrode track was visualized by coating the electrode surface with DiO (Invitrogen, Carlsbad, CA, USA). The brain was perfused with $4 \%$ paraformaldehyde (PFA), cryoprotected, and then sectioned coronally with a cryotome (40 or $60 \mu \mathrm{m}$ thickness). Sections were incubated in $0.1 \mathrm{M}$ phosphatebuffered saline (PBS) containing Triton X-100 (Sigma-Aldrich, Buchs, Switzerland) and fluorescent Nissl (1:200, NeuroTrace, N-21482 or N-21483, Invitrogen) for Nissl staining. Sections were mounted on a slide glass and embedded in an antifade solution (Dako Fluorescence Mounting Medium, S3023, Agilent, Santa Clara, CA, USA). Areal borders were determined based on fluorescent Nissl signal and a standard atlas $^{42}$. The fluorescent Nissl staining was also performed for virusinjected cortex (Supplementary Fig. 5o: N-21482 Invitrogen, Fig. 2b and Fig. 4: N21483). The image was taken with an Axio Imager Z2 (Carl Zeiss Microscopy, Jena, Germany). The objective lens was $5 \times(\mathrm{NA}=0.13)$. The filter cube for $\mathrm{DiO}$ contained an excitation bandpass filter of $470 / 40$, a dichroic filter of 495 , and an emission bandpass filter of 525/50. The filter cube for red-fluorescent Nissl contained an excitation bandpass filter of 550/25, a dichroic filter of 570, and an emission bandpass filter of 605/70. The filter cube for deep-red fluorescent Nissl contained an excitation band filter of 640/30, a dichroic filter of 660, and an emission bandpass filter of 690/50.

Three-dimensional reconstruction. In one experiment, we reconstructed 23 serial sections $(60 \mu \mathrm{m}$ thickness; every other section; $\sim 2.6 \mathrm{~mm})$ to depict the areal border of the secondary motor cortex in a microstimulated brain (Supplementary Figure 5f). First, we manually realigned each slice using MATLAB and then reconstructed serial sections with an Image J plug-in, 3D viewer. We similarly made a reconstruction for serial sections in Paxinos and Franklin's atlas ${ }^{42}$. The dorsal view of the atlas was based on the projection of the 3D reconstruction (Supplementary Figure $5 \mathrm{c}-\mathrm{e}$, Fig. $4 \mathrm{~h})$. In the image of Fig. $4 \mathrm{~h}$, the anterior-posterior level of the injection site $(0.7 \mathrm{~mm}$ anterior from the bregma) was estimated using Gaussian fitting of the tdTomato signal.

Optogenetic inhibition. Optogenetic inhibition was accomplished by selectively activating PV+interneurons locally in PV-Cre mice (Fig. 2) ${ }^{30,31}$. Following headpost implantation and craniotomy, AAV2/1-EF1 $\alpha$-DIO-hChR2(H134R)-EYFP was injected into the $\mathrm{MO}_{\mathrm{s}}$ unilaterally (4-6 sites; $60 \mathrm{nl} /$ site; depth, 200-300 $\mu \mathrm{m}$; expression $\sim 1 \mathrm{~mm}$ ) and a coverglass was placed (see "Cranial window surgery" section). A similar virus injection was performed $1 \mathrm{~mm}$ lateral to the $\mathrm{MO}_{\mathrm{s}}$ for control experiments to inhibit the primary motor cortex. Photostimulation of ChR2 was achieved using a blue laser ( $473 \mathrm{~nm}$, CrystaLaser) coupled to an optic fiber (M15L02, Thorlabs). The output power was controlled by combining a halfwave plate with a polarizing beamsplitter cube. An optical chopper was used to convert the continuous wave into a $40-\mathrm{Hz}$ pulse $\mathrm{s}^{31}$ (pulse width, $2.5 \mathrm{~ms}$; Edmund optics, 59-894). The output of the optic fiber and the surface of the cortex were placed on conjugate planes using two convex lenses ${ }^{40}(5 \mathrm{~cm}$ focal lengths). A beam splitter was placed in the infinity space, and the reflected light was focused onto the sensor of a CMOS camera (Thorlabs). This design enabled monitoring the precise location of the stimulated site on the surface of the cortex. Optogenetic inhibition was applied in $25-60 \%$ of the trials, where a particular direction of eye movements was required. The direction of eye movements was randomized among mice. The average power of the light at the surface of the cortex was $600-1200 \mu \mathrm{W}$. The effect of optogenetic inhibition was quantified to compare the probability of eye movements within $1 \mathrm{~s}$ after the target onset between trials with and without the $1 \mathrm{~s}$ laser illumination (Person's chi-square test)

Cell-attached recording. Cell-attached recording was performed to confirm the effect of optogenetic inhibition (Fig. 2c). A small craniotomy was made ( $1 \mathrm{~mm}$ diameter), and then a patch pipette was advanced blindly while monitoring its impedance to achieve a loose-seal cell-attached recording. The recording pipette contained HEPES-based artificial cerebrospinal fluid. The signal was amplified with a patch clamp amplifier (MultiClamp 700B, Molecular Devices). Signals two standard deviations larger than baseline were considered spikes. Eight cells with broad spike width were recorded.

Two-photon imaging. In vivo imaging was performed using a two-photon microscope based on a movable objective microscope system (Sutter) controlled by the ScanImage software ${ }^{66}$, as previously described ${ }^{13,67}$. The light source was a pulsed Ti:sapphire laser (Chameleon, Coherent), and the laser wavelength was set at $980 \mathrm{~nm}$, which causes a high fluorescent change in GCaMP signal ("Two-Photon Fluorescent Probes", https://www.janelia.org/lab/harris-lab-apig/research/ photophysics/two-photon-fluorescent-probes) and less scattering in the tissue than shorter wavelengths. The objective lens was apochromatic (16x, 0.80 NA, Nikon) Red and green fluorescent photons were separated using a 565-nm dichroic mirror (Chroma, 565dcxr) and barrier filters (green, ET525/70 m-2p; red, ET605/70 m2p). Signals were collected using photomultiplier tubes (Hamamatsu Photonics, H10770PA-40); frame scanning (frame rate $6-30 \mathrm{~Hz}$ ) was used. Images were collected at a depth of 150-300 $\mu \mathrm{m}$ from the dural surface for layer $2 / 3$ neurons, 75-100 $\mu \mathrm{m}$ for axonal boutons ${ }^{10}$, or $450-550 \mu \mathrm{m}$ for layer $5 \mathrm{a}$ neurons, as previously described $^{67}$. For layer 5 imaging, the back aperture of the objective was underfilled, and a larger craniotomy was made.

Image analysis. Movement artifacts were corrected for in two steps ${ }^{61}$ : a cross correlation-based image alignment (Turboreg) ${ }^{68}$ followed by a line-by-line correction using an algorithm based on a hidden-Markov model ${ }^{69}$. The regions of interest (ROIs) containing the neurons or axonal boutons were then drawn manually (3787 ROIs for $\mathrm{MO}_{\mathrm{s}}$ layer 2/3, 2321 for $\mathrm{V}_{\mathrm{RL} / \mathrm{A} / \mathrm{AL}}$ layer 2/3, 531 for $\mathrm{V} 1$ layer 2/3, 2316 for the $\mathrm{MO}_{\mathrm{s}}$ layer 5, 1199 for $\mathrm{V}_{\mathrm{RL} / \mathrm{A} / \mathrm{AL}}$ layer $2 / 3,1577$ for axonal boutons from the $\mathrm{MO}_{\mathrm{s}}$, and 760 for axonal boutons from $\mathrm{V}_{\mathrm{RL} / \mathrm{A} / \mathrm{AL}}$ ). The pixel values within each ROI were summed to estimate the fluorescence of the individual neurons/axonal boutons. $\Delta F / F$ signal was calculated as $\left(F-F_{\text {baseline }}\right) / F_{\text {baseline, }}$ where $F_{\text {baseline }}$ is the baseline fluorescence signal (30th percentile) within each trial.

Boutons from the same axons were excluded based on correlation analysis among pairs ${ }^{10,18,36,70}$. The correlation coefficients of $\triangle F / F$ during the intertrial interval were calculated for boutons in each plane, and pairs showing a higher correlation $(>0.65)$ were considered to be from the same axon. The high correlation pairs were grouped into clusters ${ }^{10,18,36,70}$, and the ROI with the largest $\Delta F / F$ signal in each cluster was assigned to represent the cluster.

For each neuron or axonal bouton, visual and motor activity were evaluated. Visual activity was quantified as an increase in $\Delta F / F$, comparing the average $\Delta F / F$ between the control range $(-150 \pm 150 \mathrm{~ms}$ from the onset of the visual stimulus) and the signal range $(200 \pm 100 \mathrm{~ms})$. Similarly, motor activity was quantified as an increase in $\Delta F / F$, comparing the average $\Delta F / F$ between the control range $(-500 \pm$ $150 \mathrm{~ms}$ from the onset of the eye movements) and the signal range $(-50 \pm 100 \mathrm{~ms})$ (rise time ${ }^{32}$ of GCaMP6f was taken into account). Neurons or boutons were considered to contain visual response if the visual activity was significantly larger than baseline activity (Wilcoxon signed-rank test; $p<0.05$ ) in either contraversive or ipsiversive movement conditions. For each neuron, preference for contraversive or ipsiversive conditions was evaluated (Mann-Whitney test; $p<0.05$ ). The motor activity was similarly evaluated. To temporally separate visual and motor activity, we rewarded mice for performing with longer reaction times during the training (Supplementary Fig. 8) and analyzed trials with reaction times $>1.5 \mathrm{~s}$.

To present the neural activity in the population (heatmaps in Fig. 3e, f, and Fig. $5 c, f)$, neurons showing a significant response either to the visual target or the motor onset were included and realigned in descending order. The activity for each neuron was normalized by its maximum $\Delta F / F$ between $0.5 \mathrm{~s}$ before the visual target onset and $0.5 \mathrm{~s}$ after the movement onset in the two target conditions.

To quantify the preference for visual and motor information (Fig. $5 \mathrm{~g}-\mathrm{l}$, Fig. $6 c-h, k-p)$, the stronger response between contraversive and ipsiversive conditions was chosen for visual and motor activity in each neuron and axon terminal and then scatter-plotted for visual ( $x$-axis) and motor ( $y$-axis) activity. The angle for each point was computed in polar representation, indicating 0 degrees for pure visual activity and 90 degrees for pure motor activity. Similarly the preference for motor information and post-movement activity was quantified (Supplementary Fig. 15). Post-movement activity was evaluated by comparing the average $\Delta F / F$ between the control range $(-500 \pm 150 \mathrm{~ms}$ from the onset of eye movements) and the signal range $(400 \pm 100 \mathrm{~ms})$.

Measurement and analysis of whisker movements. The whiskers of three mice were filmed with a CMOS camera (FL3-U3-13Y3M-C, Point Grey) controlled by the custom-made software (written in Visual C++ with the FlyCapture Software Development Kit). Images of the whiskers were saved at 66.6 or $100 \mathrm{~Hz}$ with precise frame timing at a pixel resolution of $15.4 \times 15.4 \mu \mathrm{m}^{2}$.

The movement of the whisker was measured by comparing two neighboring frames using a custom-written program in MATLAB. In the series of the images, a small ROI elongated in the anterior-posterior direction containing multiple whiskers was selected. The cross-correlation between neighboring frames was computed for the ROI, and the peak position of the correlation was considered the amount of movement of the whisker position between the two frames (Supplementary Fig. 3).

The relationship between whisking and task structure was evaluated as follows. First, frames with whisker movement $>200 \mu \mathrm{m}$ were identified. If frames were separated by $<200 \mathrm{~ms}$, all time points in between were considered the whisking period. The probability of whisking across trials was computed by aligning to the onset of the visual target or eye movement. The probability was compared between the period of $-150 \pm 150 \mathrm{~ms}$ (baseline) and $200 \pm 100 \mathrm{~ms}$ following the visual target onset and between $-500 \pm 150$ and $-50 \pm 100 \mathrm{~ms}$ before the onset of eye movements. 
Anatomical tracing. In the first experiment ( $n=3$ mice, Supplementary Fig. 6), the subcortical projection patterns of neurons in the $\mathrm{MO}_{\mathrm{s}}$ were visualized by labeling their axon terminals. A small hole $(\sim 250 \mu \mathrm{m})$ was made in the skull above the $\mathrm{MO}_{\mathrm{s}}$ ( $0.7 \mathrm{~mm}$ lateral and 0.7 rostral to the bregma), through which $\sim 50 \mathrm{nl}$ of AAV2/1EFl $\alpha$-tdTomato was injected at 300 and $800 \mu \mathrm{m}$ depths. A few weeks later, the mice were deeply anesthetized $(0.3 \mathrm{mg} / \mathrm{g}$ ketamine and $0.024 \mathrm{mg} / \mathrm{g}$ xylazine, intraperitoneally) and intracardinally perfused with $4 \%$ PFA in $0.1 \mathrm{M}$ PBS. The brains were removed, post-fixed in $4 \% \mathrm{PFA}$ at $4{ }^{\circ} \mathrm{C}$ overnight, and rinsed with $0.1 \mathrm{M}$ PBS. The brain was subsequently frozen to be sectioned coronally with a cryotome $(40 \mu \mathrm{m}$ in thickness). Sections were incubated in $0.1 \mathrm{M}$ PBS containing $4 \%$ sucrose, $0.05 \%$ $3,3^{\prime}$-diaminobenzidine, and $0.05 \%$ cytochrome oxidase at $35^{\circ} \mathrm{C}$ for $6-8 \mathrm{~h}$. Then the sections were placed on slides in mounting solution (VECTASHIELD HardSet, H1500 , Vector Laboratories). Nuclei borders were drawn based on the signal of cytochrome oxidase reactivity.

In the second experiment ( $n=3$ mice, Fig. $4 \mathrm{a}-\mathrm{d}$ ), the locations of axonal projection patterns from the $\mathrm{MO}_{\mathrm{s}}$ were identified with the help of callosal axon terminals originating from the contralateral visual area ${ }^{39-41}$. A small hole $(\sim 250$ $\mu \mathrm{m})$ was made in the skull above the right $\mathrm{MO}_{s}$, through which $\sim 50 \mathrm{nl}$ of AAV2/1EF1 $\alpha$-tdTomato was injected at 300 and $800 \mathrm{~mm}$ depths. Then a $5 \mathrm{~mm}$ square of the left cortex was exposed extending between the midline, lambda, and bregma, and 25-30 injections $\mathrm{s}^{39}$ of $\sim 40 \mathrm{nl}$ of AAV2/1-CAG-GFP were performed at a 300 $\mu \mathrm{m}$ depth. A few weeks later, the mice were perfused and the entire brains were imaged with a fluorescence microscope (Olympus MVX-10 Macroview, 1× MVX Plan Apochromat lens). The filter cube for GFP contained an excitation bandpass filter of 470/40, a dichroic filter of 495 , and an emission bandpass filter of 525/50. The filter cube for tdTomato contained an excitation bandpass filter of 545/25, a dichroic filter of 565, and an emission bandpass filter of 605/70. In some cases, the brain regions around the $\mathrm{MO}_{\mathrm{s}}$ and visual cortex were also imaged using a twophoton microscope with a low-magnification object lens (CFI Plan Fluor $4 \times$, Nikon).

In the third experiment ( $n=3$ mice, Fig. $4 \mathrm{e}-\mathrm{o}$ ), the reciprocal connectivity between the $\mathrm{MO}_{\mathrm{s}}$ and the visual cortex was examined by sequential virus injections. AAV2/1-EF1 $\alpha$-tdTomato $(\sim 50 \mathrm{nl})$ was injected into the right $\mathrm{MO}_{\mathrm{s}}$ (at 300 and 800 $\mu \mathrm{m}$ depths) through a small hole $(\sim 250 \mu \mathrm{m})$ in the skull. After a few weeks, the red signals from the axon terminals could be identified in the visual cortex using a surgical microscope modified with a custom-build light source and filters. Under the guidance of the fluorescence signal from tdTomato, AAV2/1-CAG-eGFP (40 nl) was injected at the axon terminals from the $\mathrm{MO}_{\mathrm{s}}$ in the visual cortex (depth, $250 \mu \mathrm{m})$. A few weeks later, the mice were perfused and the brain was imaged as in the second experiment. The modified surgical microscope had a $530 \mathrm{~nm}$ LED (M530L3, Thorlabs) as the excitation light source, with a short pass filter in front of it (575 nm, 84-709, Edmond Optics) and a barrier filter (610 nm, FGL610S, Thorlabs) in front of the objective lens.

Quantification of anatomical projections. The strength of the anatomical connections between the frontal and parieto-occipital cortex was evaluated using an available database from the Allen Institute for Brain Science ${ }^{7}$ (Supplementary Fig. 12). To quantify projections from the frontal to parieto-occipital cortex, we first set a search filter (source structure: $\mathrm{MO}_{\mathrm{s}}$ or $\mathrm{ACA}_{\mathrm{d}}$ ) to collect available datasets and then downloaded these as a comma-separated values file. We selected the experiments for which the tracer injection site was (1) at 0.5-1.0 mm lateral and $0.5-1.0 \mathrm{~mm}$ rostral from the bregma $\left(\mathrm{MO}_{\mathrm{s}}\right.$, experiment numbers: 141603190 , $168162771,287995889,266503540,272822110,277957202$, and 297892130); (2) at 0-0.5 mm lateral and rostral $\left(\mathrm{ACA}_{\mathrm{d}}\right.$, experiment numbers: 125833030,478491090 , 496576666, 478376911, 158018630, 287599685, 526783054, and 177783204); or (3) at $1.0-1.5 \mathrm{~mm}$ lateral and rostral $\left(\mathrm{MO}_{\mathrm{s}}\right.$, experiment numbers: 181598954 ,

$266176167,141602484,288169842,183617432,167569313,168002073,297669605$, 267750528,156492394 , and 267659565 , presumed the whisker motor area ${ }^{71}$ ). Then, we checked axonal density values at 'VISa', 'VISrl', 'VISal', 'VISp', or 'SSbfd' (corresponding to areas A, RL, AL, V1, and S1 barrel cortex, respectively) for each experiment, normalized them with the largest density value in each experiment, and then compared them. Experiments in (2) were excluded if the injection contained $>30 \%$ of $\mathrm{MO}_{\mathrm{s}}$ to reduce contamination. Experiments with interneuron Cre, layer $4 \mathrm{Cre}$, or layer 6 Cre lines, and those with a labeled sum value $<1$ were also excluded because long-range projections were rare.

To evaluate projections from the parieto-occipital to the frontal cortex, we similarly set the source structure (VISa, VISrl, VISal, VISp, and SSbfd) to collect available datasets. The datasets were injection (4) at VISa, VISrl, or VISal $(n=19$ experiments, experiment numbers: 524874308, 518606617, 540145406, 521600943, 303616127, 522635991, 272929308, 518741580, 517325325, 518605900, 120437703, 524666904, 528509838, 297231636, 267761438, 294533406, 510834706, 520996382, 510848595); (5) at VISp ( $n=12$ experiments, experiment numbers: 309113907, 307320960, 307593747, 530574594, 479755622, 307557934, 309003780, 535692871, 531441947, 482578964, 501117182, and 266250195, contamination outside VISp < $2 \%)$; and (6) at barrel cortex ( $n=9$ experiments, experiment numbers: 112951804, $100142655,127866392,112882565,297654263,159602992,272735030,100141473$, and 175819113 , contamination outside SSp-bfd $<50 \%$ ). Animals with a labeled sum value $<1$ (1.5 for VISp) were excluded. We then measured axonal projection density for each experiment using coronal sections available at Allen's website. First, the sections $1.5 \mathrm{~mm}$ rostral to the bregma (rostrolateral $\mathrm{MO}_{s}$, presumed whisker motor area), $0.75 \mathrm{~mm}\left(\mathrm{MO}_{\mathrm{s}}\right)$, or $0.25 \mathrm{~mm}\left(\mathrm{ACA}_{\mathrm{d}}\right)$ were downloaded. Then axonal density was quantified with Image J; ROIs were drawn to cover the $\mathrm{MO}_{\mathrm{s}}$ or $\mathrm{ACA}_{\mathrm{d}}$, which matched Allen's reference atlas shown next to the section, and the mean gray value for each ROI was measured. Finally, each value was normalized with the largest density value in each experiment and then compared with each other.

Immunohistochemistry. PV was immunostained using standard procedures (Fig. 2b). Coronal slices (thickness, $40 \mu \mathrm{m}$ ) were cut using a cryostat and blocked in carrier solution (5\% bovine serum albumin (Sigma-Aldrich); 0.3\% Triton X-100 (Sigma-Aldrich) in $0.1 \mathrm{M}$ PBS) for $2 \mathrm{~h}$ at room temperature on a shaker. Slices were then incubated with anti-PV primary antibody (1:5000 in carrier solution; Cat. \#24428, Hudson, WI, USA) for $18 \mathrm{~h}$ at $4{ }^{\circ} \mathrm{C}$ on a shaker. After three rinses with 0.1 M PBS for $30 \mathrm{~min}$, sections were incubated with Alexa-Fluor-568-conjugated goat anti-guinea pig secondary antibody (A11075, Invitrogen, 1:500 in carrier solution) for $1 \mathrm{~h}$ at room temperature on a shaker. After a few additional rinses for $30 \mathrm{~min}$ in $0.1 \mathrm{M}$ PBS, slices were mounted on slide glasses for imaging.

Statistical analysis. All statistical tests were non-parametric and are indicated in the main text or figure legend. Sample sizes were larger than the minimum size that can yield $p<0.05$ for each test. Data and traces were show as mean \pm s.e.m., unless otherwise stated. Power calculations to predetermine sample sizes were not performed, but our sample sizes are similar to those reported in previous publications ${ }^{67,72}$.

Code availability. The computer codes from this study are available from the corresponding author upon reasonable request.

Data availability. The data for this study are available from the corresponding author upon reasonable request.

Received: 24 February 2017 Accepted: 5 December 2017 Published online: 23 January 2018

\section{References}

1. Brodmann, K. Beitrage zur histologischen localisation der grosshirnrinde. Dritte mitteilung. Die rindenfelder der niederen affen. J. Psychol. Neurol. 4, 177-226 (1905)

2. Felleman, D. J. \& Van Essen, D. C. Distributed hierarchical processing in the primate cerebral cortex. Cereb. Cortex 1, 1-47 (1991).

3. Wise, S. P., Boussaoud, D., Johnson, P. B. \& Caminiti, R. Premotor and parietal cortex: corticocortical connectivity and combinatorial computations. Annu. Rev. Neurosci. 20, 25-42 (1997).

4. Markov, N. T. \& Kennedy, H. The importance of being hierarchical. Curr. Opin Neurobiol. 23, 187-194 (2013).

5. Andermann, M. L., Kerlin, A. M., Roumis, D. K., Glickfeld, L. L. \& Reid, R. C. Functional specialization of mouse higher visual cortical areas. Neuron 72, 1025-1039 (2011).

6. Marshel, J. H., Garrett, M. E., Nauhaus, I. \& Callaway, E. M. Functional specialization of seven mouse visual cortical areas. Neuron 72, 1040-1054 (2011).

7. Oh, S. W. et al. A mesoscale connectome of the mouse brain. Nature $\mathbf{5 0 8}$, 207-214 (2014).

8. Zingg, B. et al. Neural networks of the mouse neocortex. Cell 156, 1096-1111 (2014).

9. Glickfeld, L. L., Andermann, M. L., Bonin, V. \& Reid, R. C. Cortico-cortical projections in mouse visual cortex are functionally target specific. Nat. Neurosci. 16, 219-226 (2013)

10. Kwon, S. E., Yang, H., Minamisawa, G. \& O'Connor, D. H. Sensory and decision-related activity propagate in a cortical feedback loop during touch perception. Nat. Neurosci. 19, 1243-1249 (2016).

11. Yamashita, T. et al. Membrane potential dynamics of neocortical projection neurons driving target-specific signals. Neuron 80, 1477-1490 (2013).

12. Chen, J. L., Carta, S., Soldado-Magraner, J., Schneider, B. L. \& Helmchen, F. Behaviour-dependent recruitment of long-range projection neurons in somatosensory cortex. Nature 499, 336-340 (2013).

13. Sato, T. R. \& Svoboda, K. The functional properties of barrel cortex neurons projecting to the primary motor cortex. J. Neurosci. 30, 4256-4260 (2010).

14. Ferezou, I. et al. Spatiotemporal dynamics of cortical sensorimotor integration in behaving mice. Neuron 56, 907-923 (2007).

15. Xu, N. L. et al. Nonlinear dendritic integration of sensory and motor input during an active sensing task. Nature 492, 247-251 (2012).

16. Huber, D. et al. Multiple dynamic representations in the motor cortex during sensorimotor learning. Nature 484, 473-478 (2012).

17. Matyas, F. et al. Motor control by sensory cortex. Science 330, 1240-1243 (2010). 
18. Petreanu, L. et al. Activity in motor-sensory projections reveals distributed coding in somatosensation. Nature 489, 299-303 (2012).

19. Zagha, E., Casale, A. E., Sachdev, R. N., McGinley, M. J. \& McCormick, D. A. Motor cortex feedback influences sensory processing by modulating network state. Neuron 79, 567-578 (2013).

20. Douglas, R. J., Koch, C., Mahowald, M., Martin, K. A. \& Suarez, H. H. Recurrent excitation in neocortical circuits. Science 269, 981-985 (1995).

21. Murphy, B. K. \& Miller, K. D. Balanced amplification: a new mechanism of selective amplification of neural activity patterns. Neuron 61, 635-648 (2009).

22. Friston, K. The free-energy principle: a unified brain theory? Nat. Rev. Neurosci. 11, 127-138 (2010).

23. Hanes, D. P. \& Schall, J. D. Neural control of voluntary movement initiation. Science 274, 427-430 (1996).

24. Carpenter, R. H. S. Movements of the Eyes (Pion Limited, London, 1988).

25. Van Gisbergen, J. A., Robinson, D. A. \& Gielen, S. A quantitative analysis of generation of saccadic eye movements by burst neurons. J. Neurophysiol. 45, 417-442 (1981)

26. Bahill, A. T., Clark, M. R. \& Stark, L. The main sequence, a tool for studying human eye movements. Math. Biosci. 24, 191-204 (1975).

27. Sakatani, T. \& Isa, T. PC-based high-speed video-oculography for measuring rapid eye movements in mice. Neurosci. Res. 49, 123-131 (2004).

28. Collewijn, H., Erkelens, C. J. \& Steinman, R. M. Binocular co-ordination of human horizontal saccadic eye movements. J. Physiol. 404, 157-182 (1988).

29. Zhang, S. et al. Long-range and local circuits for top-down modulation of visual cortex processing. Science 345, 660-665 (2014).

30. Olsen, S. R., Bortone, D. S., Adesnik, H. \& Scanziani, M. Gain control by layer six in cortical circuits of vision. Nature 483, 47-52 (2012).

31. Cardin, J. A. et al. Driving fast-spiking cells induces gamma rhythm and controls sensory responses. Nature 459, 663-667 (2009).

32. Chen, T. W. et al. Ultrasensitive fluorescent proteins for imaging neuronal activity. Nature 499, 295-300 (2013).

33. Bruce, C. J. \& Goldberg, M. E. Primate frontal eye fields. I. Single neurons discharging before saccades. J. Neurophysiol. 53, 603-635 (1985)

34. Bruce, C. J., Goldberg, M. E., Bushnell, M. C. \& Stanton, G. B. Primate frontal eye fields. II. Physiological and anatomical correlates of electrically evoked eye movements. J. Neurophysiol. 54, 714-734 (1985).

35. Schlag, J. \& Schlag-Rey, M. Evidence for a supplementary eye field. J. Neurophysiol. 57, 179-200 (1987).

36. Roth, M. M. et al. Thalamic nuclei convey diverse contextual information to layer 1 of visual cortex. Nat. Neurosci. 19, 299-307 (2016).

37. Mohajerani, M. H. et al. Spontaneous cortical activity alternates between motifs defined by regional axonal projections. Nat. Neurosci. 16, 1426-1435 (2013).

38. Murakami, T., Yoshida, T., Matsui, T. \& Ohki, K. Wide-field $\mathrm{Ca}(2+)$ imaging reveals visually evoked activity in the retrosplenial area. Front. Mol. Neurosci. 8, 20 (2015).

39. Wang, Q. \& Burkhalter, A. Area map of mouse visual cortex. J. Comp. Neurol. 502, 339-357 (2007)

40. Sato, T. K., Hausser, M. \& Carandini, M. Distal connectivity causes summation and division across mouse visual cortex. Nat. Neurosci. 17, 30-32 (2014).

41. Wang, Q., Sporns, O. \& Burkhalter, A. Network analysis of corticocortical connections reveals ventral and dorsal processing streams in mouse visual cortex. J. Neurosci. 32, 4386-4399 (2012).

42. Paxinos, G. \& Franklin, K. The Mouse Brain in Stereotaxic Coordinates 2nd edn (Academic Press, San Diego, 2001).

43. Mishkin, M. \& Ungerleider, L. G. Contribution of striate inputs to the visuospatial functions of parieto-preoccipital cortex in monkeys. Behav. Brain. Res. 6, 57-77 (1982).

44. Zhang, S. et al. Organization of long-range inputs and outputs of frontal cortex for top-down control. Nat. Neurosci. 19, 1733-1742 (2016).

45. Sreenivasan, V., Kyriakatos, A., Mateo, C., Jaeger, D. \& Petersen, C. C. Parallel pathways from whisker and visual sensory cortices to distinct frontal regions of mouse neocortex. Neurophotonics 4, 031203 (2017).

46. Koch, C. \& Reid, R. C. Neuroscience: observatories of the mind. Nature 483, 397-398 (2012).

47. Sakatani, T. \& Isa, T. Quantitative analysis of spontaneous saccade-like rapid eye movements in C57BL/6 mice. Neurosci. Res. 58, 324-331 (2007).

48. Fuller, J. H. Eye and head movements in the pigmented rat. Vision Res. 25, 1121-1128 (1985).

49. Hikosaka, O. \& Sakamoto, M. Dynamic characteristics of saccadic eye movements in the albino rat. Neurosci. Res. 4, 304-308 (1987).

50. Wang, L., Liu, M., Segraves, M. A. \& Cang, J. Visual experience is required for the development of eye movement maps in the mouse superior colliculus. $J$. Neurosci. 35, 12281-12286 (2015).
51. Wallace, D. J. et al. Rats maintain an overhead binocular field at the expense of constant fusion. Nature 498, 65-69 (2013).

52. Huerta, M. F., Krubitzer, L. A. \& Kaas, J. H. Frontal eye field as defined by intracortical microstimulation in squirrel monkeys, owl monkeys, and macaque monkeys. II. Cortical connections. J. Comp. Neurol. 265, 332-361 (1987).

53. Erlich, J. C., Bialek, M. \& Brody, C. D. A cortical substrate for memory-guided orienting in the rat. Neuron 72, 330-343 (2011).

54. Manita, S. et al. A top-down cortical circuit for accurate sensory perception. Neuron 86, 1304-1316 (2015).

55. Schall, J. D. \& Thompson, K. G. Neural selection and control of visually guided eye movements. Annu. Rev. Neurosci. 22, 241-259 (1999).

56. Zirnsak, M. \& Moore, T. Saccades and shifting receptive fields: anticipating consequences or selecting targets? Trends Cogn. Sci. 18, 621-628 (2014).

57. Rigotti, M. et al. The importance of mixed selectivity in complex cognitive tasks. Nature 497, 585-590 (2013).

58. Raposo, D., Kaufman, M. T. \& Churchland, A. K. A category-free neural population supports evolving demands during decision-making. Nat. Neurosci. 17, 1784-1792 (2014).

59. Hardcastle, K., Maheswaranathan, N., Ganguli, S. \& Giocomo, L. M. A multiplexed, heterogeneous, and adaptive code for navigation in medial entorhinal cortex. Neuron 94, 375-387 (2017). e377.

60. Hippenmeyer, S. et al. A developmental switch in the response of DRG neurons to ETS transcription factor signaling. PLoS Biol. 3, e159 (2005).

61. Komiyama, T. et al. Learning-related fine-scale specificity imaged in motor cortex circuits of behaving mice. Nature 464, 1182-1186 (2010).

62. Sato, T., Murthy, A., Thompson, K. G. \& Schall, J. D. Search efficiency but not response interference affects visual selection in frontal eye field. Neuron $\mathbf{3 0}$ 583-591 (2001).

63. Sato, T. R. \& Schall, J. D. Effects of stimulus-response compatibility on neural selection in frontal eye field. Neuron 38, 637-648 (2003).

64. Canny, J. A computational approach to edge detection. IEEE Trans. Pattern Anal. Mach. Intell. 8, 679-698 (1986).

65. Neafsey, E. J. et al. The organization of the rat motor cortex: a microstimulation mapping study. Brain Res. 396, 77-96 (1986).

66. Pologruto, T. A., Sabatini, B. L. \& Svoboda, K. ScanImage: flexible software for operating laser scanning microscopes. Biomed. Eng. Online 2, 13 (2003).

67. Hasegawa, M. et al. Selective suppression of local circuits during movement preparation in the mouse motor cortex. Cell Rep. 18, 2676-2686 (2017).

68. Thevenaz, P., Ruttimann, U. E. \& Unser, M. A pyramid approach to subpixel registration based on intensity. IEEE Trans. Image Process. 7, 27-41 (1998).

69. Dombeck, D. A., Khabbaz, A. N., Collman, F., Adelman, T. L. \& Tank, D. W Imaging large-scale neural activity with cellular resolution in awake, mobile mice. Neuron 56, 43-57 (2007).

70. Sun, W., Tan, Z., Mensh, B. D. \& Ji, N. Thalamus provides layer 4 of primary visual cortex with orientation- and direction-tuned inputs. Nat. Neurosci. 19, 308-315 (2016).

71. Sreenivasan, V. et al. Movement initiation signals in mouse whisker motor cortex. Neuron 92, 1368-1382 (2016).

72. Peters, A. J., Chen, S. X. \& Komiyama, T. Emergence of reproducible spatiotemporal activity during motor learning. Nature 510, 263-267 (2014)

73. Tohmi, M., Meguro, R., Tsukano, H., Hishida, R. \& Shibuki, K. The extrageniculate visual pathway generates distinct response properties in the higher visual areas of mice. Curr. Biol. 24, 587-597 (2014).

74. Takahashi, T. The organization of the lateral thalamus of the hooded rat. J. Comp. Neurol. 231, 281-309 (1985).

\section{Acknowledgements}

We thank S. Kashiwagi for technical help; K. Svoboda, H.P. Thier for discussions; Y. Dan, B. Haider, T. Imai, and M. Izumo for reading the manuscript; and L.L. Looger, K. Svoboda, J. Akerboom, D.S. Kim, and the GENIE Project at Janelia Farm for making GCaMP6 available. This work was supported by grants from the Japan Science and Technology Agency (PRESTO) and DFG (SA 2575/2-1, SA 2575/3-1) to T.R.S. and the Werner Reichardt Centre for Integrative Neuroscience (CIN) at the Eberhard Karls University of Tübingen. The CIN is an Excellence Cluster funded by the Deutsche Forschungsgemeinschaft within the framework of the Excellence Initiative (EXC 307). T K.S. was supported by the Japan Society for the Promotion of Science Postdoctoral Fellowship for Research Abroad.

\section{Author contributions}

T.R.S. and T.K.S. conceived the study. T.R.S. designed and developed the task. T.Itokazu. M.H., R.K., H.O., K.S., T.K.S. and T.R.S. performed behavioral training, electrical stimulation, optogenetic suppression, and calcium imaging. M.H., T.Itokazu., H.O., T.Ito., U.-R.A. and S.C. performed anatomical experiments. T.K.S., T.Itokazu., R.K., H.I. 
and T.R.S. analyzed the data. T.Itokazu., T.K.S. and T.R.S. wrote the paper with inputs from M.H.

\section{Additional information}

Supplementary Information accompanies this paper at https://doi.org/10.1038/s41467017-02501-4.

Competing interests: The authors declare no competing financial interests.

Reprints and permission information is available online at http://npg.nature.com/ reprintsandpermissions/

Publisher's note: Springer Nature remains neutral with regard to jurisdictional claims in published maps and institutional affiliations. (c) (i) Open Access This article is licensed under a Creative Commons Attribution 4.0 International License, which permits use, sharing, adaptation, distribution and reproduction in any medium or format, as long as you give appropriate credit to the original author(s) and the source, provide a link to the Creative Commons license, and indicate if changes were made. The images or other third party material in this article are included in the article's Creative Commons license, unless indicated otherwise in a credit line to the material. If material is not included in the article's Creative Commons license and your intended use is not permitted by statutory regulation or exceeds the permitted use, you will need to obtain permission directly from the copyright holder. To view a copy of this license, visit http://creativecommons.org/ licenses/by/4.0/.

(C) The Author(s) 2018 